\title{
Platelet-Rich Plasma in Maxillary Sinus Augmentation: Systematic Review
}

\author{
Holmes Ortega-Mejia ${ }^{1}$, Albert Estrugo-Devesa ${ }^{2} ®$, Constanza Saka-Herrán ${ }^{1}$, \\ Raúl Ayuso-Montero ${ }^{2}$, José López-López ${ }^{2,3, *}$ and Eugenio Velasco-Ortega ${ }^{4}$ \\ 1 Faculty of Medicine and Health Sciences (Dentistry), University of Barcelona, 08970 Barcelona, Spain; \\ holmes.ortegamejia@gmail.com (H.O.-M.); constanzasakah@gmail.com (C.S.-H.) \\ 2 Oral Health and Masticatory System Group (Bellvitge Biomedical Research Institute) IDIBELL, Department \\ of Odontostomatology, Faculty of Medicine and Health Sciences (Dentistry), University of Barcelona, \\ 08970 Barcelona, Spain; albertestrugodevesa@gmail.com (A.E.-D.); raulayuso@ub.edu (R.A.-M.) \\ 3 Service of the Medical-Surgical Area of Dentistry Hospital, University of Barcelona, 08970 Barcelona, Spain \\ 4 Department of Stomatology, Faculty of Dentistry, University of Seville, 41013 Seville, Spain; evelasco@us.es \\ * Correspondence: jl.lopez@ub.edu; Tel.: +34-93-402-42-71; Fax: +34-93-402-42-48
}

Received: 7 January 2020; Accepted: 28 January 2020; Published: 30 January 2020

\begin{abstract}
Background: Sinus augmentation can be performed with or without grafting biomaterials, and to date, there is no quality evidence regarding the augmentation of the sinus floor using only platelet concentrates, which can improve the healing period and enhance bone regeneration by stimulating angiogenesis and bone formation. The main objective of this paper was to assess the effect of the sole use of platelet concentrates in sinus augmentation in terms of newly formed bone, augmented bone height, and clinical outcomes and to assess the additional beneficial effects of platelet-rich fibrin (PRF) in combination with other grafting biomaterials. Methods: A systematic review was conducted following Preferred Reporting Items for Systematic reviews and Meta-Analyses (PRISMA) guidelines. Pooled analyses were performed with the Review Manager software. Results: For sinus elevation only using platelet concentrates, 11 studies met the inclusion criteria and were included for qualitative synthesis. Only one study was a clinical trial, which reported improved outcomes for the allograft group compared to the titanium-PRF (T-PRF) group. A total of 12 studies where PRF was used in addition to grafting biomaterials met eligibility criteria and were included in the review. Results from meta-analyses provided no additional beneficial effects of PRF in sinus augmentation in terms of bone height and percentage of soft tissue area. There was a statistically significant lower percentage of residual bone substitute material in the PRF (+) group compared to the PRF (-) group. The percentage of newly formed bone was slightly higher in the PRF (+) group, but this was not statistically significant. Conclusion: There is no robust evidence to make firm conclusions regarding the beneficial effects of the sole use of platelet concentrates in sinus augmentation. However, studies have shown favorable outcomes regarding implant survival, bone gain, and bone height. The use of PRF with other grafting biomaterials appears to provide no additional beneficial effects in sinus lift procedures, but they may improve the healing period and bone formation. Well-conducted randomized clinical trials (RCTs) are necessary to confirm the available results to provide recommendations for the clinical practice.
\end{abstract}

Keywords: platelet-rich plasma; platelet-rich fibrin; bone substitutes; maxillary sinus augmentation; sinus floor augmentation 


\section{Introduction}

\section{Rationale}

Among other objectives, implant dentistry seeks to restore the loss of dental and supportive structures, either because of an aesthetic and/or functional problem for the patient [1]. For this reason, the concept of osseointegration, introduced by Branemark in the 1950s, and the use of dental implants for prosthetic purposes has become a widely spread treatment option [2].

One of the big challenges that arise from the deficiency of hard and soft tissues is represented in atrophic maxilla [3]. Implant placement may be compromised due to the pneumatisation of the maxillary sinus, with subsequent posterior alveolar bone loss and lack of bone quality and/or quantity [4]. Hence, several treatment options have been developed during the last 60 years, but it is since the 1980s that the augmentation of the maxillary sinus has become a surgical treatment option. Although not exempt from complications [5], it improves the condition of the implant recipient site in the upper-posterior zone [6-12].

There are different surgical approaches to elevate the Schneiderian membrane in order to achieve the vertical height of the alveolar process required for implant placement with an adequate length $[13,14]$. These techniques include the lateral window approach, which was first developed by Tatum [7] in the mid-1970s and later described by Boyne and James in 1980 [6]. Tatum first suggested the transalveolar technique for sinus floor elevation in 1986 [7], which was later modified by Summers [15] in 1994. This modified approach is called the osteotome technique, and it uses a set of osteotomes of increasing diameters intended to increase the density of the soft maxillary bone and create an upward fracture of the maxillary sinus floor.

The sinus membrane elevation can be performed with or without the use of grafting materials [16]. The non-use of grafting materials with an immediate placement of implants was initially introduced by Lundgren et al [17]; it requires a sufficient residual vertical alveolar bone height in the posterior zone of the maxilla to achieve an adequate primary stability of the inserted implant necessary to preserve the augmented Schneiderian membrane, thus allowing a clot formation around the exposed surface of the implant in the sinus cavity. This approach has been reported in several systematic reviews including, mostly, short-term descriptive studies [18-20].

On the other hand, there are different grafting materials commonly used to elevate the maxillary sinus floor, including autograft, allograft, xenograft, alloplast, and growth factors [21,22]. The selection of the ideal grafting material has been a matter of controversy over the years. However, due to its osteoinductive, osteogenic, and osteoconductive properties, autogenous bone graft is considered the gold standard [23-25]. Autologous bone graft has some inconveniences, the most important ones being its greater morbidity and unpredictable reabsorption [26-28]. This has led to an increase in the use of biological and synthetic bone substitutes in order to simplify the surgical procedure and to avoid the aforementioned complications. All these approaches are well documented in numerous systematic reviews and meta-analyses [29-34].

Platelet concentrates, as initially described by Whitman et al. [35] are blood-derived products obtained after the centrifugation of a blood sample. Different techniques have been developed to obtain a variety of preparations. To obtain platelet-rich plasma (PRP) and plasma rich in growth factors (PRGF), citrated blood is used during the centrifugation process to prevent coagulation, resulting in liquid PRP and PRGF. For its gel form, thrombin and/or calcium chloride is added to induce fibrin polymerization creating a low-density fibrin gel preparation. For platelet-rich fibrin (PRF), blood is collected without any anticoagulant, and it is immediately centrifugated, during which the natural coagulation process occurs [36,37]. The use of autologous platelet concentrates in the different fields of dentistry started in the 1980-1990s. Since then, platelet concentrates and growth factors have been used to enhance guided tissue regeneration in periodontology, in the regeneration of the pulpodentin complex, and in the guided restoration of bone loss in oral surgery [38]. 
To date, there is no high-quality evidence regarding the augmentation of the sinus floor using only PRP, PRGF, or PRF. The combination of PRP and autologous bone for sinus lift seems to provide no additional beneficial effects in terms of implant survival rate, implant stability, bone height, marginal bone level, bone density, laminar bone and tissue volume, bone graft resorption, angiogenesis, and soft tissue healing, compared to the use of only autologous bone, as reviewed by Strauss et al. in 167 patients [39]. Another review, which analysed 81 patients, reported a short-term improvement in densitometry values and new bone formation, but its clinical relevance is unclear [40]. The use of PRP in combination with Beta-Tricalcium Phosphate ( $\beta-\mathrm{TCP})$, which was studied in 35 patients [30], or xenografts, in 127 patients [41,42], appears to show no additional clinical benefits. The addition of PRF to deproteinized bovine bone mineral (DBBM) has also not shown further benefits [30,41,42]. However, in vivo animal studies have shown that the sole use of platelet concentrates in sinus augmentation achieves a mean height of newly formed bone of up to $3.6 \mathrm{~mm}[43,44]$, and recent clinical studies have reported that they could be a successful procedure in sinus augmentation on residual ridges $<5 \mathrm{~mm} \mathrm{[45]} \mathrm{and} \mathrm{achieve} \mathrm{a} \mathrm{mean} \mathrm{height} \mathrm{of} \mathrm{newly} \mathrm{bone} \mathrm{of} \mathrm{up} \mathrm{to} 4 \mathrm{~mm}$ when implants are simultaneously placed $[46,47]$.

\section{Objectives}

The main objective was to assess the effect of the sole use of platelet concentrates in maxillary sinus augmentation in terms of newly formed bone, augmented bone height, and clinical outcomes such as implant stability and implant survival. Furthermore, to evaluate the additional effects of PRF in combination with other graft materials in sinus lift relative to the outcomes mentioned above, we conducted a systematic review that answered the following structure question (PICO):

$\mathrm{P}=$ Patients requiring unilateral or bilateral maxillary sinus augmentation

$\mathrm{I}=$ Sole use of platelet concentrates / PRF + grafting materials

$\mathrm{C}=$ Grafting materials or nothing

$\mathrm{O}=$ Newly formed bone, augmented bone height, implant stability and implant survival

\section{Methods}

\subsection{Eligibility Criteria}

The review included articles that met the following inclusion criteria:

a. Patients in need of unilateral or bilateral sinus augmentation before implant dental placement.

b. Randomized Clinical Trials (RCT), Controlled Clinical Trials (CCT), and comparative studies assessing histological, histomorphometric, clinical, and radiographic outcomes on the additional effects of PRF in sinus augmentation versus the non-use of PRF.

c. Specified follow-up period.

As the available literature on the effects of the sole use of platelet concentrates in sinus augmentation is scarce in terms of evidence provided by RCT and CCT, we considered including retrospective studies, prospective studies, and case series. Case series including fewer than five patients were excluded. In vitro and in vivo (animal) studies were also excluded from this review.

\subsection{Information Sources}

The electronic search was conducted in MEDLINE (PuBMed) and the Cochrane Central Register for Controlled Trials. No restriction on date publication was applied and only articles published in English were considered. References of relevant studies selected for potential inclusion were also searched. The last search was performed on 9 December 2019. 


\subsection{Search Strategy}

For the MEDLINE (PubMed) search, the following search terms were used:

i- Sole use of platelet concentrates in sinus floor augmentation:

("Platelet rich fibrin"(All Fields) OR "platelet rich plasma" (All Fields) OR "PRP" (All Fields) OR "PRF" (All Fields) OR "PRGF" (All Fields) AND "sinus floor" (All Fields))

ii- PRF + grafting biomaterials in sinus floor augmentation:

("Sinus Floor Augmentation" (Mesh) OR "sinus lifting" (All Fields)) AND ("Platelet-Rich Fibrin" (Mesh) OR (platetet-rich (All Fields) AND ("plasma" (MeSH Terms) OR "plasma" (All Fields))) OR (("plasma" (MeSH Terms) OR "plasma" (All Fields)) AND rich (All Fields) AND ("intercellular signaling peptides and proteins" (MeSH Terms) OR ("intercellular" (All Fields) AND "signaling" (All Fields) AND "peptides" (All Fields) AND "proteins" (All Fields)) OR "intercellular signaling peptides and proteins" (All Fields) OR ("growth" (All Fields) AND "factors" (All Fields)) OR "growth factors" (All Fields])))

\subsection{Study Selection}

Three authors independently reviewed the titles and abstracts of the references located in the databases. Articles were selected whenever they appeared to meet the inclusion criteria. The same two authors independently reviewed the studies selected in the initial screening for full-text revision and final selection. Disagreements were resolved by discussion. If no consensus between the two authors could be achieved, a third reviewer was consulted.

\subsection{Data Collection Process and Items}

Two reviewers extracted the following data from the selected studies: year of publication, country, design, study period, follow-up, number of patients, number of sinuses, sinus lift complications, implant surgery, number of implants, intervention, and comparison group, which were arranged in a table. Outcome measures were arranged in another table, which included: radiographic, histomorphometric, clinical and postoperative complications assessment. Two authors revised the data collection.

\subsection{Outcomes and Summary Measures}

Primary outcomes were the percentage of newly formed bone, percentage of residual-bone substitute material, and percentage of soft tissue area assessed by histomorphometric analyses. Furthermore, augmented bone height $(\mathrm{mm})$ was assessed by radiographic evaluation and clinical outcomes in terms of implant stability (implant stability quotient), implant survival (\%), and postoperative complications.

\subsection{Risk of Bias in Individual Studies}

For RCT, the authors critically appraised each study for potential risk of bias by the Cochrane Collaboration's tool for assessing risk of bias. Studies were classified as "low risk", "unclear risk", and "high risk" of bias.

\subsection{Synthesis of Results}

Pooled analyses were performed using a random-effects model. Heterogeneity between studies was assessed by the $\mathrm{I}^{2}$ statistics. Heterogeneity was considered statistically significant for a $\mathrm{p}$ value $<0.1$. Heterogeneity was interpreted as recommended by the Cochrane Handbook: $0 \%-40 \%$ was considered unimportant, $30 \%-60 \%$ was considered as moderate heterogeneity, $50 \%-90 \%$ was considered as substantial heterogeneity, and $75 \%-100 \%$ was considered as considerable heterogeneity. Outcomes were combined using the Review Manager software, version 5.3 (Cochrane community, available in: https://community.cochrane.org/help/tools-and-software/revman-5/revman-5-download; accessed in 1 November 2019) 


\section{Results}

\subsection{Study Selection}

The search among the literature for the sole use of platelet concentrates on maxillary sinus augmentation yielded 132 studies, which were screened for eligibility. After title and abstract evaluation, 18 studies were selected for full-text review. Of these, seven studies failed to meet inclusion criteria and were excluded from the review. Reasons for exclusion were in vivo (animal studies) $[43,44,48]$, case series with $<5$ patients [49-51], and the use of a platelet concentrate in combination with a graft material [52]. Finally, 11 studies were included for qualitative synthesis (Figure 1A). For the use of PRF in combination with other grafting materials, the search yielded 74 articles. After duplicates were removed, 62 articles were screened for eligibility based on their title and abstract, from which 21 were selected for full-text review. Eleven studies were excluded, as they did not meet the inclusion criteria, nine studies were excluded because they lacked a comparison group [53-61], one was excluded because it was compared with patients treated for another reason [62], and another was excluded because the intervention was not of interest for this study [63]. Additionally, two articles were identified through other sources and were incorporated in the review. Finally, 12 studies were included for qualitative synthesis (Figure 1B).

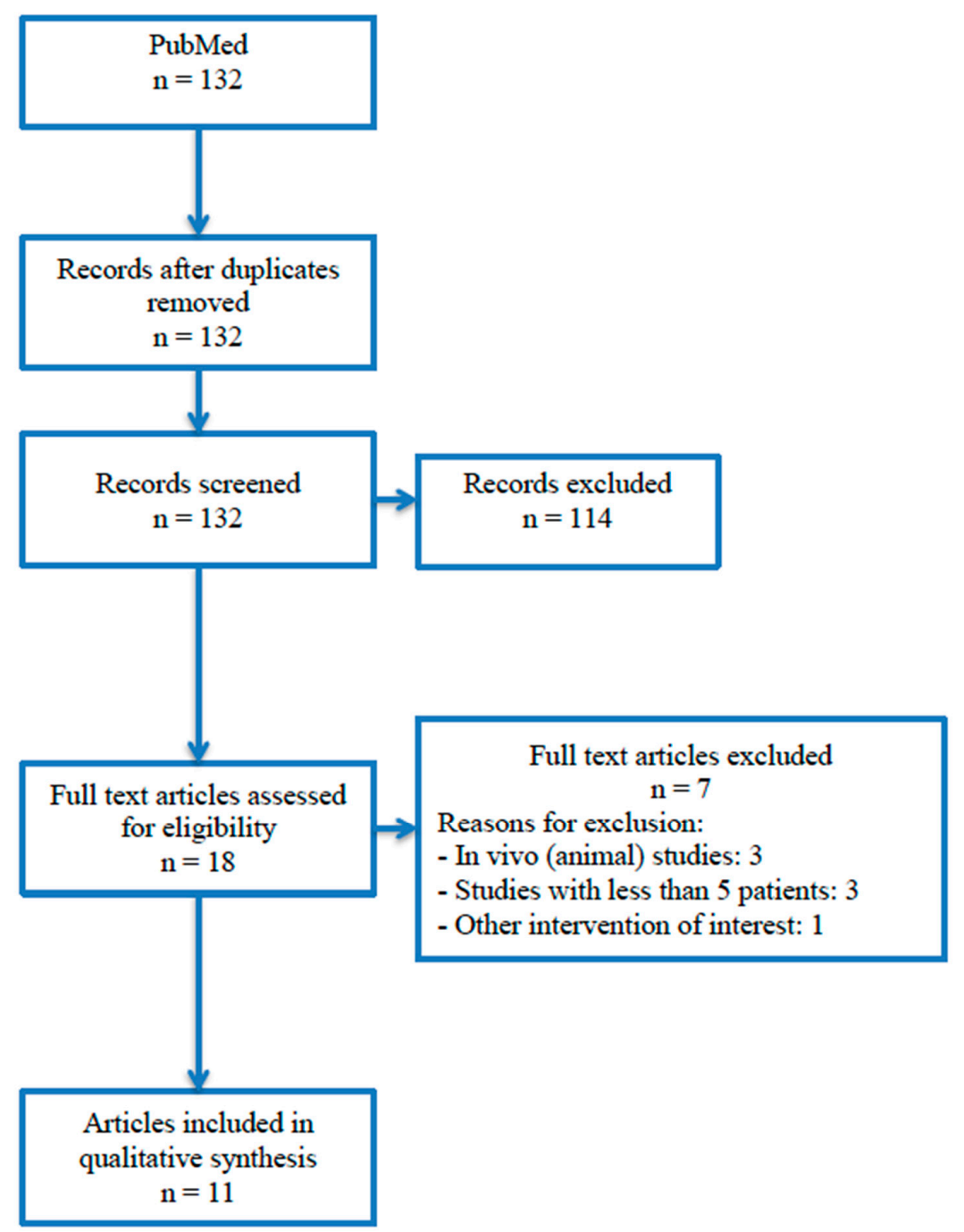

(A)

Figure 1. Cont. 


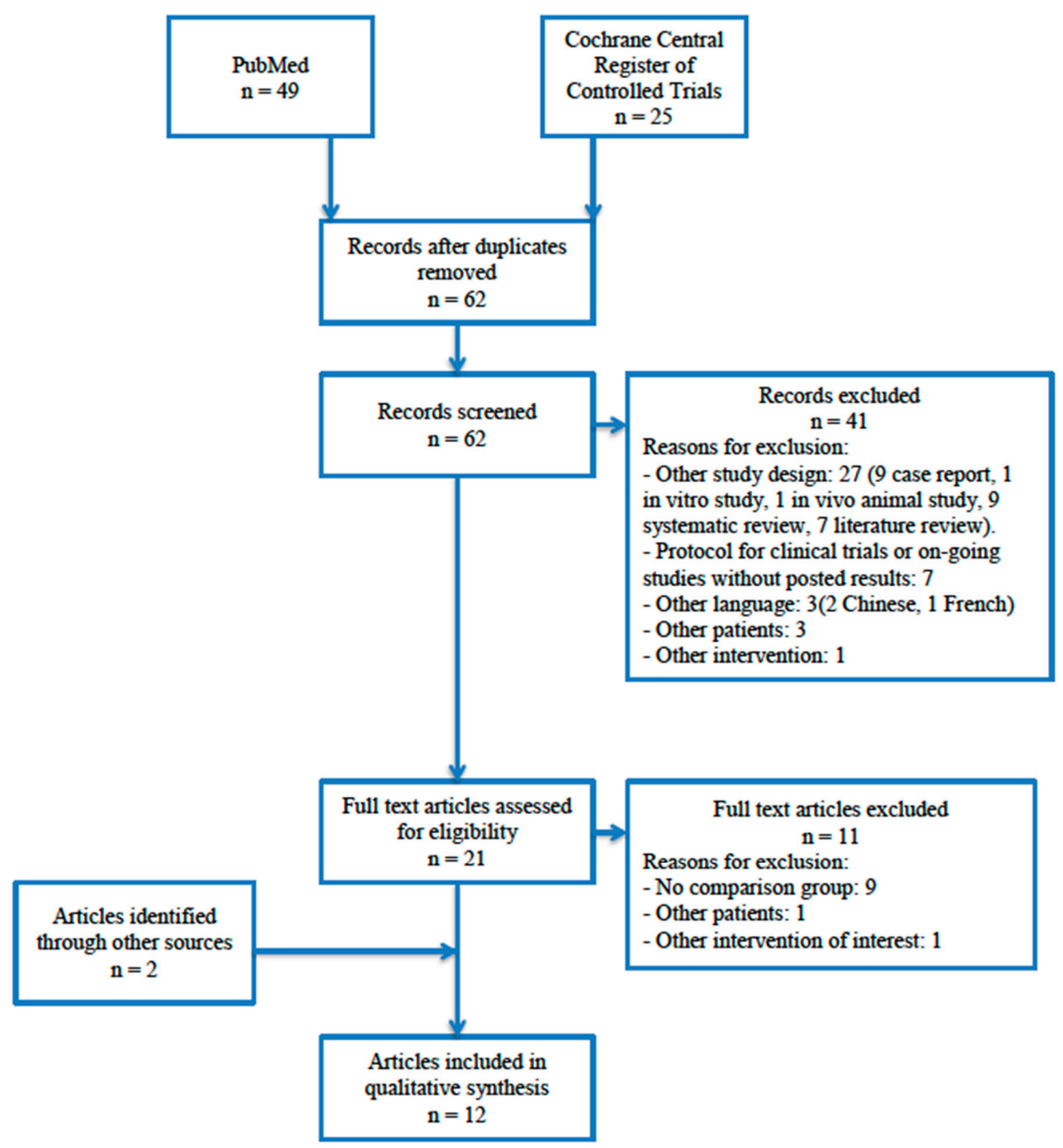

(B)

Figure 1. (A) Diagram for study selection: sole use of platelet concentrates in sinus floor augmentation. (B) Diagram for study selection: platelet-rich fibrin (PRF) in combination with other grafting biomaterials in sinus floor augmentation.

\subsection{Study Characteristics}

\subsubsection{Sole Use of Platelet Concentrates in Sinus Floor Augmentation:}

Among the 11 included studies, two were case series [58,60], three were retrospective studies [53,64,65], five were prospective studies [46,47,66-68], and one was an RCT [45]. The minimum follow-up was six months with a maximum of six years. In total, studies involved 301 patients requiring maxillary sinus elevation and 223 requiring sinuses lift procedures. Five studies did not report on the number of sinus elevations [46,53,64-66]. A total of 481 implants were placed, all with an immediate protocol. The majority of the studies used PRF as the sole grafting material. The descriptive characteristics of the studies are shown in Table 1. The only RCT [45] had a follow-up of nine months, involving 18 patients, from whom 10 were allocated to the intervention group (titanium-PRF) and the others were allocated to the control group (Cells \& Tissue Bank Austria, Cells \& Tissue Bank Austria (CTBA) allograft). There were 18 maxillary sinus elevations, with no reported complications and 37 implant placements in a delayed protocol (Table 2). 
Table 1. Descriptive characteristics of studies included for sole use of platelet concentrates in sinus floor augmentation.

\begin{tabular}{|c|c|c|c|c|c|c|c|c|c|}
\hline $\begin{array}{l}\text { Author, Year } \\
\text { Country }\end{array}$ & $\begin{array}{c}\text { Design } \\
\text { Study Period } \\
\text { Follow-Up }\end{array}$ & $\begin{array}{c}\mathrm{N}^{\circ} \\
\text { Patients }\end{array}$ & $\begin{array}{l}\text { Gender } \\
\text { Age }\end{array}$ & $N^{o}$ Sinus & $\begin{array}{l}\text { Sinus Lift } \\
\text { Complications }\end{array}$ & $\begin{array}{c}\text { Implant } \\
\text { Surgical } \\
\text { Stage }\end{array}$ & $\begin{array}{c}\mathrm{N}^{\circ} \\
\text { Implants }\end{array}$ & $\begin{array}{c}\text { Platelet } \\
\text { Concentrate }\end{array}$ & Outcome Measures \\
\hline $\begin{array}{l}\text { Anitua et al, } 2016 \\
\text { Spain [53] }\end{array}$ & $\begin{array}{l}\text { Retrospective } \\
\text { Study } \\
3 \text { years }\end{array}$ & 26 & $\begin{array}{c}\text { F: } 14 \\
\text { M: } 12 \\
55 \pm 7 y\end{array}$ & NR & NR & $\begin{array}{l}\text { Immediate } \\
\text { placement }\end{array}$ & 41 & $\begin{array}{l}\text { PRGF } \\
\text { PRGF + bone } \\
\text { graft in } 7 \\
\text { implants }\end{array}$ & $\begin{array}{l}\text { Radiographic assessment }(\text { mean } \pm \mathrm{sd}){ }^{*} \\
\text { Bone height (1year): } 8.2 \pm 1.2 \mathrm{~mm} \\
\text { Bone height ( } 3 \text { years): } 8.8 \pm 1.4 \mathrm{~mm} \\
\text { Bone gain }(1 \text { year): } 3.6 \pm 1.8 \mathrm{~mm} \\
\text { Bone gain (3 years): } 4.3 \pm 2.0 \mathrm{~mm}\end{array}$ \\
\hline $\begin{array}{l}\text { Aoki et al, } 2018 \\
\text { Japan [64] }\end{array}$ & $\begin{array}{l}\text { Retrospective } \\
\text { Study } \\
2010-2015 \\
X=3.43 \text { years } \\
(1-7 \text { y) }\end{array}$ & 34 & $\begin{array}{l}\text { F: } 17 \\
\text { M: } 17 \\
57.6 y\end{array}$ & NR & NR & $\begin{array}{l}\text { Immediate } \\
\text { placement }\end{array}$ & 71 & PRF & $\frac{\text { Clinical assessment }}{\text { Implant survival: } 85.5 \%}$ \\
\hline $\begin{array}{l}\text { Diss et al, } 2008 \\
\text { France [66] }\end{array}$ & $\begin{array}{l}\text { Prospective } \\
\text { Study } \\
2004-2005 \\
1 \text { year }\end{array}$ & 20 & $\begin{array}{c}\text { F: } 14 \\
\text { M: } 6 \\
54.8 \pm 11.1 \mathrm{y}\end{array}$ & NR & $\begin{array}{c}4 \\
\text { (Sinus } \\
\text { membrane } \\
\text { perforation) }\end{array}$ & $\begin{array}{l}\text { Immediate } \\
\text { placement }\end{array}$ & 35 & PRF & $\begin{array}{l}\text { Radiographic assessment }(\text { mean } \pm \text { sd }) \\
\text { Bone gain: } 3.2 \pm 1.5 \mathrm{~mm}(0.1-5.8 \mathrm{~mm}) \\
\text { Clinical assessment } \\
\text { Implant survival: } 97.1 \%\end{array}$ \\
\hline $\begin{array}{l}\text { Gulsen et al, } 2019 \\
\text { Turkey [65] }\end{array}$ & $\begin{array}{l}\text { Retrospective } \\
\text { Study } \\
2015-2018 \\
6 \text { months }\end{array}$ & 12 & $\begin{array}{c}\text { F: } 7 \\
\text { M: } 5 \\
55.7 \pm 8.3\end{array}$ & NR & $\begin{array}{c}1 \\
\text { (Sinus } \\
\text { membrane } \\
\text { perforation) }\end{array}$ & $\begin{array}{l}\text { Immediate } \\
\text { placement }\end{array}$ & 18 & $\begin{array}{l}\text { i-PRF soaked } \\
\text { collagen }\end{array}$ & $\begin{array}{l}\text { Radiographic assessment }(\text { mean } \pm \mathrm{sd}) \\
\text { Bone height: } 11.6 \pm 1.6 \mathrm{~mm}(8-14 \mathrm{~mm}) \\
\text { Bone gain: } 6.3 \pm 1.3 \mathrm{~mm}(4.2-8.5 \mathrm{~mm}) \\
\text { Clinical assessment } \\
\text { Implant survival: } 100 \%\end{array}$ \\
\hline $\begin{array}{c}\text { Kanayama et al, } \\
2016 \\
\text { Japan [46] }\end{array}$ & $\begin{array}{l}\text { Prospective } \\
\text { Study } \\
2011-2013 \\
1 \text { year }\end{array}$ & 27 & $\begin{array}{c}\text { F: } 15 \\
\text { M: } 12 \\
54.2 y \\
(29-74 y)\end{array}$ & NR & 0 & $\begin{array}{l}\text { Immediate } \\
\text { placement }\end{array}$ & 39 & PRF & $\begin{array}{l}\text { Radiographic assessment }(\text { mean } \pm \text { sd }) \\
\text { Bone gain: } \\
\text { SA implant: } 4.4 \pm 1.7 \mathrm{~mm}(\mathrm{p}<0.001) \\
\text { HA implant: } 4.0 \pm 1.6 \mathrm{~mm} \\
\text { Clinical assessment } \\
\text { Implant survival: } 100 \%\end{array}$ \\
\hline $\begin{array}{l}\text { Mazor et al, } 2009 \\
\text { Israel [58] }\end{array}$ & $\begin{array}{l}\text { Case series } \\
2007-2008 \\
6 \text { months }\end{array}$ & 20 & $\begin{array}{c}\text { F: } 14 \\
\text { M: } 6 \\
54.1 \pm 5.2 y\end{array}$ & 25 & 0 & $\begin{array}{l}\text { Immediate } \\
\text { placement }\end{array}$ & 41 & L-PRF & $\begin{array}{l}\text { Radiographic assessment }(\text { mean } \pm \text { sd }) \\
\text { Bone gain: } 10.1 \pm 0.9 \mathrm{~mm}(7-13 \mathrm{~mm}) \\
\text { Histomorphometric assessment }(\text { mean } \pm \mathrm{sd}) \\
\text { Newly formed bone: } 33 \% \pm 5 \% \\
\text { Clinical assessment } \\
\text { Implant survival: } 100 \%\end{array}$ \\
\hline
\end{tabular}


Table 1. Cont.

\begin{tabular}{|c|c|c|c|c|c|c|c|c|c|}
\hline $\begin{array}{l}\text { Author, Year } \\
\text { Country }\end{array}$ & $\begin{array}{c}\text { Design } \\
\text { Study Period } \\
\text { Follow-Up }\end{array}$ & $\begin{array}{c}\mathrm{N}^{\mathrm{o}} \\
\text { Patients }\end{array}$ & $\begin{array}{l}\text { Gender } \\
\text { Age }\end{array}$ & $\mathbf{N}^{\circ}$ Sinus & $\begin{array}{l}\text { Sinus Lift } \\
\text { Complications }\end{array}$ & $\begin{array}{c}\text { Implant } \\
\text { Surgical } \\
\text { Stage }\end{array}$ & $\begin{array}{c}\mathrm{N}^{\circ} \\
\text { Implants }\end{array}$ & $\begin{array}{c}\text { Platelet } \\
\text { Concentrate }\end{array}$ & Outcome Measures \\
\hline $\begin{array}{c}\text { Molemans et al, } \\
2019 \\
\text { Belgium [67] }\end{array}$ & $\begin{array}{l}\text { Prospective } \\
\text { Study } \\
2015-2016 \\
6 \text { months }\end{array}$ & 26 & $\begin{array}{c}\text { F: } 12 \\
\text { M: } 14 \\
55 y(38-78)\end{array}$ & 28 & NR & $\begin{array}{l}\text { Immediate } \\
\text { placement }\end{array}$ & 29 & L-PRF & $\begin{array}{l}\text { Radiographic assessment (mean } \pm \text { sd) } \\
\text { Bone gain: } \\
\text { TSFE: } 3.4 \pm 1.2 \mathrm{~mm} / / \text { LSFE: } 5.4 \pm 1.5 \mathrm{~mm} \\
\text { Clinical assessment } \\
\text { Implant survival: } 93.1 \% \text { ( } 2 \text { implants were not } \\
\text { osseointegrated and were removed) }\end{array}$ \\
\hline $\begin{array}{c}\text { Simonpieri et al, } \\
2011 \\
\text { France [60] }\end{array}$ & $\begin{array}{c}\text { Case series } \\
2003-2008 \\
6 \text { years }\end{array}$ & 20 & $\begin{array}{c}\text { F: } 12 \\
\text { M: } 8 \\
59.8 \pm 11.1 y\end{array}$ & 23 & $\begin{array}{c}3 \\
\text { (Sinus } \\
\text { membrane } \\
\text { perforation) }\end{array}$ & $\begin{array}{l}\text { Immediate } \\
\text { placement }\end{array}$ & 52 & L-PRF & $\begin{array}{l}\text { Radiographic assessment }(\text { mean } \pm \mathrm{sd}) \\
\text { Bone gain: } 10.4 \pm 1.2 \mathrm{~mm}(8.5-12 \mathrm{~mm}) \\
\text { Clinical assessment } \\
\text { Implant survival: } 100 \%\end{array}$ \\
\hline $\begin{array}{l}\text { Tajima et al, } \\
2013 \\
\text { Japan [47] }\end{array}$ & $\begin{array}{l}\text { Prospective } \\
\text { Study } \\
2009-2011 \\
6 \text { months }\end{array}$ & 6 & $\begin{array}{c}\text { F: } 6 \\
67.8 \mathrm{y} \\
(53-82 y)\end{array}$ & 9 & 0 & $\begin{array}{l}\text { Immediate } \\
\text { placement }\end{array}$ & 17 & PRF & $\begin{array}{l}\text { Radiographic assessment }(\text { mean } \pm s d) \\
\text { Bone height: } 11.8 \pm 1.7 \mathrm{~mm}(9.1-14.1 \mathrm{~mm}) \\
\text { Bone gain: } 7.5 \mathrm{~mm} \\
\text { Bone density: } 323 \pm 156.2 \mathrm{HU}(185-713) \\
\text { Bone volume: } 0.7 \pm 0.3 \mathrm{~mL}\end{array}$ \\
\hline $\begin{array}{c}\text { Toffler et al, } \\
2010 \\
\text { USA [68] }\end{array}$ & $\begin{array}{l}\text { Prospective } \\
\text { Study } \\
2008-2010 \\
11 \text { months }\end{array}$ & 110 & $\begin{array}{l}\text { F: } 70 \\
\text { M: } 40 \\
58.4 \text { y } \\
(34-90 y)\end{array}$ & 138 & $\begin{array}{c}5 \\
\text { (Sinus } \\
\text { membrane } \\
\text { perforation) }\end{array}$ & $\begin{array}{l}\text { Immediate } \\
\text { placement }\end{array}$ & 138 & PRF & $\begin{array}{l}\text { Radiographic assessment }(\text { mean } \pm \mathrm{sd}) \\
\text { Bone gain: } 3.4 \mathrm{~mm}(3.5-5 \mathrm{~mm}) \\
\text { Clinical assessment } \\
\text { Implant survival: } 96.4 \%\end{array}$ \\
\hline
\end{tabular}

Abbreviations = F: Female; M: Male; y: year; PRGF: Plasma rich in growth factors; NR: not reported; i-PRF: injectable platelet-rich fibrin; HA: hydroxyapatite; SA: sandblasted acid-etched;

L-PRF: leukocyte-platelet rich fibrin; TSFE: transalveolar sinus floor elevation; LSFE: lateral window sinus floor elevation. * Results for sole use of PRGF (34 implants). 
Table 2. Descriptive characteristics of studies included for the use of PRF combined with other grafting materials in sinus floor augmentation.

\begin{tabular}{|c|c|c|c|c|c|c|c|c|}
\hline $\begin{array}{l}\text { Author, } \\
\text { Year } \\
\text { Country }\end{array}$ & $\begin{array}{l}\text { Design// } \\
\text { Study Period } \\
\text { Follow Up }\end{array}$ & $\mathrm{N}^{\circ}$ Patients & $\begin{array}{c}\mathbf{N}^{\circ} \\
\text { Sinuses }\end{array}$ & $\begin{array}{c}\text { Sinus Lift } \\
\text { Complications }\end{array}$ & $\begin{array}{l}\text { Implant } \\
\text { Surgery }\end{array}$ & $\begin{array}{c}\mathrm{N}^{\mathrm{o}} \\
\text { Implants }\end{array}$ & $\begin{array}{l}\text { Intervention Group } \\
\text { (I) }\end{array}$ & $\begin{array}{l}\text { Control Group } \\
\text { (C) }\end{array}$ \\
\hline $\begin{array}{c}\text { Bolukbasi et al, } \\
2015 \\
\text { Turkey [69] }\end{array}$ & $\begin{array}{l}\text { Retrospective } \\
\text { Study } \\
2008-2012 \\
2 \text { years }\end{array}$ & 25 & $\begin{array}{l}\text { I: } 17 \\
\text { C: } 15\end{array}$ & $\begin{array}{l}\text { I: } 0 \\
\text { C: } 0\end{array}$ & $\begin{array}{l}6 \text { months after } \\
\text { sinus lifting }\end{array}$ & $\begin{array}{l}\text { I: } 34 \\
\text { C: } 32\end{array}$ & $\begin{array}{l}\text { Bovine bone graft material } \\
\text { (Bio-Oss) + PRF mixture }\end{array}$ & $\begin{array}{l}\text { Bovine bone graft material } \\
\text { (Bio-Oss) + collagen membrane } \\
\text { (Bio-Gide) }\end{array}$ \\
\hline $\begin{array}{l}\text { Bosshardt et al, } \\
2014 \\
\text { Switzerland [71] }\end{array}$ & $\begin{array}{l}\text { Controlled Trial } \\
7-11 \text { months }\end{array}$ & $\begin{array}{l}8 \\
\text { I: } 5 \\
\text { C: } 3\end{array}$ & $\begin{array}{l}12 \\
\text { I: } 8 \\
\text { C: } 4\end{array}$ & $\begin{array}{l}\text { I: } 0 \\
\text { C: } 0\end{array}$ & $\begin{array}{l}\text { 7-11 months } \\
\text { after sinus } \\
\text { lifting }\end{array}$ & 16 & $\begin{array}{l}\text { Synthetic nanocrystalline } \\
\text { hydroxyapatite embedded in } \\
\text { highly porous silica gel matrix } \\
\text { (NanoBone) + PRF }\end{array}$ & $\begin{array}{l}\text { Synthetic nanocrystalline } \\
\text { hydroxyapatite embedded in } \\
\text { highly porous silica gel matrix } \\
\text { (NanoBone) + collagen } \\
\text { membrane (BioGide) }\end{array}$ \\
\hline $\begin{array}{l}\text { Choukroun et al, } \\
2006 \\
\text { France [70] }\end{array}$ & $\begin{array}{l}\text { Retrospective } \\
\text { study } \\
2001-2003 \\
8 \text { months }\end{array}$ & 9 & $\begin{array}{l}\text { I: } 6 \\
\text { C: } 3\end{array}$ & $\begin{array}{c}\mathrm{I}: 1 \\
\text { (Perforation of } \\
\text { the sinus } \\
\text { membrane) } \\
\text { C: } 0\end{array}$ & $\begin{array}{l}\text { I: } 4 \text { months after } \\
\text { sinus lifting } \\
\text { C: } 8 \text { months } \\
\text { after sinus } \\
\text { lifting }\end{array}$ & NR & $\begin{array}{l}\text { Freeze-dried bone } \\
\text { allograft + PRF }\end{array}$ & Freeze-dried bone allograft \\
\hline $\begin{array}{l}\text { Comert et al, } \\
2017 \\
\text { Turkey [72] }\end{array}$ & $\begin{array}{c}\text { RCT } \\
2012-2013 \\
6 \text { months }\end{array}$ & $\begin{array}{l}26 \\
\text { Group A: } 9 \\
\text { Group B: } 8 \\
\text { Control: } 9\end{array}$ & NR & $\begin{array}{c}\text { Group A: } 1 \\
\text { Group B: } 2 \\
\text { Control: } 2 \\
\text { (Perforation of } \\
\text { the sinus } \\
\text { membrane) }\end{array}$ & $\begin{array}{l}6 \text { months after } \\
\text { sinus lifting }\end{array}$ & NR & $\begin{array}{l}\text { Group A: P-PRP mixed } \beta \text { - TCP } \\
\text { Group B: PRF mixed } \beta \text { - TCP }\end{array}$ & $\beta-$ ТCP \\
\hline $\begin{array}{c}\text { Del Fabbro et al } \\
\text { 2013, } \\
\text { Italy [73] }\end{array}$ & $\begin{array}{c}\text { RCT } \\
\text { 2011-2012 } \\
\text { 1 week }\end{array}$ & $\begin{array}{l}30 \\
\text { I: } 15 \\
\text { C: } 15\end{array}$ & NR & $\begin{array}{c}\text { I: } 1 \\
\text { C: } 2 \\
\text { (Perforation of } \\
\text { the sinus } \\
\text { membrane) }\end{array}$ & $\begin{array}{l}\text { 6-8 months after } \\
\text { sinus lifting }\end{array}$ & NR & $\begin{array}{l}\text { Deproteinized bovine bone } \\
\text { matrix (Bio-Oss) + } \\
\text { PRGF-Endoret }\end{array}$ & $\begin{array}{l}\text { Deproteinized bovine bone } \\
\text { matrix (Bio-Oss) }\end{array}$ \\
\hline $\begin{array}{l}\text { Gassling et al, } \\
2013 \\
\text { Germany [74] }\end{array}$ & $\begin{array}{l}\text { Split mouth } \\
\text { RCT } \\
2010-2011 \\
5 \text { months }\end{array}$ & 6 & $\begin{array}{l}\text { I: } 6 \\
\text { C: } 6\end{array}$ & NR & $\begin{array}{l}5 \text { months after } \\
\text { sinus lifting }\end{array}$ & 32 & $\begin{array}{l}\text { Autologous bone and } \\
\text { bone-substitute material } \\
\text { (Bio-Oss) mixed in 1:1 ratio + } \\
\text { PRF }\end{array}$ & $\begin{array}{l}\text { Autologous bone and } \\
\text { bone-substitute material } \\
\text { (Bio-Oss) mixed in 1:1 ratio }+ \\
\text { collagen membrane (Bio-Gide) }\end{array}$ \\
\hline $\begin{array}{l}\text { Gurler et al. } \\
2016 \\
\text { Turkey [75] }\end{array}$ & $\begin{array}{c}\text { RCT } \\
2 \text { weeks }\end{array}$ & $\begin{array}{c}28 \\
\text { (4 excluded } \\
\text { from analyses) } \\
\text { I: } 12 \\
\text { C: } 12\end{array}$ & $\begin{array}{l}28 \\
\text { I: } 12 \\
\text { C: } 12\end{array}$ & $\begin{array}{c}4 \\
\text { (2 postoperative } \\
\text { maxillary } \\
\text { sinusitis and } 2 \\
\text { perforation of } \\
\text { the sinus } \\
\text { membrane) }\end{array}$ & NR & NR & $\begin{array}{l}\text { Allogenous freeze dried } \\
\text { corticocancellous bone chips } \\
\text { (MinerOss) + L-PRF }\end{array}$ & $\begin{array}{l}\text { Allogenous freeze dried } \\
\text { corticocancellous bone chips } \\
\text { (MinerOss) }\end{array}$ \\
\hline
\end{tabular}


Table 2. Cont

\begin{tabular}{|c|c|c|c|c|c|c|c|c|}
\hline $\begin{array}{l}\text { Author, } \\
\text { Year } \\
\text { Country }\end{array}$ & $\begin{array}{c}\text { Design/ } \\
\text { Study Period } \\
\text { Follow Up }\end{array}$ & $\mathbf{N}^{\circ}$ Patients & $\begin{array}{c}\mathbf{N}^{\circ} \\
\text { Sinuses }\end{array}$ & $\begin{array}{c}\text { Sinus Lift } \\
\text { Complications }\end{array}$ & $\begin{array}{l}\text { Implant } \\
\text { Surgery }\end{array}$ & $\begin{array}{c}\mathrm{N}^{\mathrm{o}} \\
\text { Implants }\end{array}$ & $\begin{array}{l}\text { Intervention Group } \\
\text { (I) }\end{array}$ & $\begin{array}{l}\text { Control Group } \\
\text { (C) }\end{array}$ \\
\hline $\begin{array}{l}\text { Nizam et al, } \\
2017 \\
\text { Turkey [41] }\end{array}$ & $\begin{array}{c}\text { Split mouth } \\
\text { RCT } \\
2013-2015 \\
6 \text { months } \\
\end{array}$ & 13 & $\begin{array}{l}26 \\
\text { I: } 13 \\
\text { C: } 13\end{array}$ & $\begin{array}{l}\text { I: } 0 \\
\text { C: } 0\end{array}$ & $\begin{array}{l}6 \text { months after } \\
\text { sinus lifting }\end{array}$ & $\begin{array}{l}\text { I: } 30 \\
\text { C: } 28\end{array}$ & $\begin{array}{l}\text { Deproteinized bovine bone } \\
\text { mineral (BioOss) + L-PRF }\end{array}$ & $\begin{array}{l}\text { Deproteinized bovine bone } \\
\text { mineral (BioOss) }\end{array}$ \\
\hline $\begin{array}{l}\text { Olgun et al, } \\
2018 \\
\text { Turkey [45] }\end{array}$ & $\begin{array}{c}\text { RCT } \\
\text { 2013-2014 } \\
9 \text { months }\end{array}$ & 18 & $\begin{array}{l}18 \\
\text { I: } 10 \\
\text { C: } 8\end{array}$ & $\begin{array}{l}\text { I: } 0 \\
\text { C: } 0\end{array}$ & $\begin{array}{l}\text { I: } 4 \text { months after } \\
\text { sinus lifting } \\
\text { C: } 6 \text { months } \\
\text { after sinus } \\
\text { lifting }\end{array}$ & 37 & Titanium-PRF & Allograft (CTBA Allograft) \\
\hline $\begin{array}{c}\text { Pichotano et al, } \\
2018 \\
\text { Brazil [42] }\end{array}$ & $\begin{array}{l}\text { Split mouth } \\
\text { RCT } \\
2014-2015 \\
8 \text { months }\end{array}$ & 12 & $\begin{array}{c}22 \\
\text { I: } 12 \\
\text { C: } 12\end{array}$ & $\begin{array}{l}\text { I: } 0 \\
\text { C: } 0\end{array}$ & $\begin{array}{l}\text { I: } 4 \text { months after } \\
\text { sinus lifting } \\
\text { C: } 8 \text { months } \\
\text { after sinus } \\
\text { lifting }\end{array}$ & $\begin{array}{l}\text { I: } 19 \\
\text { C: } 19\end{array}$ & $\begin{array}{l}\text { Demineralized bovine bone } \\
\text { mineral (BioOss) + L-PRF }\end{array}$ & $\begin{array}{l}\text { Demineralized bovine bone } \\
\text { mineral (BioOss) }\end{array}$ \\
\hline $\begin{array}{l}\text { Tatullo et al, } \\
2012 \\
\text { Italy [76] }\end{array}$ & $\begin{array}{c}\text { RCT } \\
150 \text { days }\end{array}$ & $\begin{array}{c}60 \\
\text { I: } 30 \\
\text { C: } 18 \\
\text { Split mouth: } \\
12\end{array}$ & 72 & $\begin{array}{l}\text { I: } 0 \\
\text { C: } 0\end{array}$ & $\begin{array}{c}\text { Early (106 days } \\
\text { after sinus lift): } \\
20 \\
\text { Intermediate } \\
\text { (120 days after } \\
\text { sinus lift): } 20 \\
\text { Late (150 days } \\
\text { after sinus lift): } \\
20\end{array}$ & 240 & $\begin{array}{l}\text { Deproteinized bovine bone } \\
\text { mineral (BioOss) + PRF }\end{array}$ & $\begin{array}{l}\text { Deproteinized bovine bone } \\
\text { mineral (BioOss) }\end{array}$ \\
\hline $\begin{array}{l}\text { Zhang et al, } \\
2012 \\
\text { China [77] }\end{array}$ & $\begin{array}{c}\text { RCT } \\
6 \text { months }\end{array}$ & 11 & $\begin{array}{l}11 \\
\text { I: } 6 \\
\text { C: } 5\end{array}$ & NR & $\begin{array}{l}6 \text { months after } \\
\text { sinus lifting }\end{array}$ & $\begin{array}{l}\text { I: } 6 \\
\text { C: } 5\end{array}$ & $\begin{array}{l}\text { Deproteinized bovine bone } \\
\text { mineral (BioOss) + PRF }\end{array}$ & $\begin{array}{l}\text { Deproteinized bovine bone } \\
\text { mineral (BioOss) }\end{array}$ \\
\hline
\end{tabular}

Abbreviations: I: Intervention group; C: Control group; PRF: Platelet-Rich Fibrin; NR: Not Reported; RCT: Randomized Clinical Trial; P-PRP: Platelet-Rich Plasma; $\beta$ - TCP: Beta-Tricalcium Phosphate; L-PRF: Leukocyte-PRF; PRGF: Plasma Rich in Growth Factors. 


\subsection{2. $P R F+$ Grafting Biomaterials in Sinus Floor Augmentation:}

Among the 12 studies, two were retrospective studies [69,70], one was a CCT [71], and nine were RCTs [41,42,45,72-77], of which three had a split mouth design [41,42,74]. Follow-up ranged from one week to two years. Studies involved 246 patients in need of unilateral or bilateral sinus floor augmentation, and a total of 298 sinus lift procedures were performed, of which 149 were allocated to the intervention group (PRF + grafting biomaterial). A total of 498 implants were placed with a delayed protocol. Only one study allocated patients in an early, intermediate, or late protocol for implant placement [76]. The majority of the studies combined PRF with bovine bone mineral (Bio-Oss) [41,42,69,73,74,76,77], while two combined PRF with alloplastic grafts [71,72], two combined PRF with bone allograft [70,75], and one used titanium-PRF as the only grafting biomaterial [45]. Summary descriptive characteristics of included studies are shown in Table 2.

\subsection{Risk of Bias within Studies}

From the 12 studies assessing the effect of PRF on sinus floor augmentation, nine were RCT. The risk of bias for these trials is presented in Table 3. Seven studies were considered as having a high risk of bias, mainly because the lack of blinding of participants and personnel $[41,45,72,73,75-77]$. These studies also did not specify their methods for allocation concealment, and some did not report the methods for random sequence generation, potentially introducing selection bias. One study was assessed as having an unclear risk of bias [74], and only one study was assessed as having a low risk of bias [42].

Table 3. Risk of Bias of included randomized clinical trials.

\begin{tabular}{|c|c|c|c|c|c|c|c|}
\hline $\begin{array}{l}\text { Randomized } \\
\text { Clinical Trials }\end{array}$ & $\begin{array}{l}\text { Random } \\
\text { Sequence } \\
\text { Generation }\end{array}$ & $\begin{array}{l}\text { Allocation } \\
\text { Concealment }\end{array}$ & $\begin{array}{c}\text { Blinding of } \\
\text { Participants, } \\
\text { Personnel }\end{array}$ & $\begin{array}{l}\text { Blinding of } \\
\text { Outcome } \\
\text { Assessment }\end{array}$ & $\begin{array}{c}\text { Incomplete } \\
\text { Outcome Data }\end{array}$ & $\begin{array}{l}\text { Selective } \\
\text { Reporting }\end{array}$ & $\begin{array}{c}\text { Other } \\
\text { Bias }\end{array}$ \\
\hline Comert et al [72] & $?$ & $?$ & - & + & + & + & + \\
\hline Gassling et al [74] & $?$ & + & $?$ & + & + & + & + \\
\hline Gurler et al [75] & + & $?$ & - & + & - & + & + \\
\hline Pichotano et al [42] & + & + & + & + & + & + & + \\
\hline Tatullo et al [76] & + & $?$ & - & $?$ & + & + & + \\
\hline Zhang et al [77] & $?$ & $?$ & - & $?$ & + & + & + \\
\hline
\end{tabular}

+: Low;?: Unclear;-: High.

\subsection{Results of Individual Studies}

Summary data of the results of included studies for the sole use of platelet concentrates on sinus floor augmentation are presented in Table 1. Results of individual studies for the use of PRF in combination with other grafting materials on sinus lift are illustrated in Table 4. 
Table 4. Summary of outcome measures of studies reviews for PRF on sinus augmentation.

\begin{tabular}{|c|c|}
\hline $\begin{array}{l}\text { Author, Year } \\
\text { Country }\end{array}$ & Outcome Measures \\
\hline $\begin{array}{l}\text { Bolukbasi et al, } \\
2015 \\
\text { Turkey [69] }\end{array}$ & $\begin{array}{l}\text { Radiographic assessment }\left(\mathrm{T}_{0}-\mathrm{T}_{5}\right)(\mathrm{mean} \pm \text { standard deviation }) \\
\text { Relationship between sinus-graft height and the implant: } \\
\text { BL/IL ratio a : Intervention group: } 1.36 \pm 0.04 / / \text { Control group: } 1.36 \pm 0.03 \\
\text { During study period, intervention group showed less change in BL/IL values than control group }(\mathrm{p}=0.022) \\
\text { Change in the height of grafted sinus: } \\
\text { GSH/OSH ratio } \mathrm{b}: \text { Intervention group: } 4.49 \pm 0.29 \text { // Control group: } 4.14 \pm 0.20(\mathrm{p}=0.093) \\
\text { Histomorphometric assessment }(\%)(\text { mean } \pm \text { standard deviation) } \\
\text { New bone formation: Intervention group: } 35 \pm 8.6 / / \text { Control group: } 32.9 \pm 9.7(\mathrm{p}=0.61) \\
\text { Connective tissue: Intervention group: } 30.6 \pm 7.5 / / \text { Control group: } 33.9 \pm 9.2(\mathrm{p}=0.34) \\
\text { Biomaterial remnants: Intervention group: } 33.1 \pm 6.3 \text { // Control group: } 33.8 \pm 8.6(\mathrm{p}=0.87)\end{array}$ \\
\hline $\begin{array}{l}\text { Bosshardt et al, } \\
2014 \\
\text { Switzerland [71] }\end{array}$ & $\begin{array}{l}\text { Histomorphometric assessment }(\%) \text { (mean } \pm \text { standard deviation) } \\
\text { New bone formation: Intervention group (PRF): } 28.6 \pm 6.9 \text { // Control group: } 28.7 \pm 5.4 \\
\text { Residual bone substitute material: Intervention group (PRF): } 25.6 \pm 8.7 / / \text { Control group: } 25.5 \pm 7.6 \\
\text { Soft Tissue: Intervention group (PRF): } 45.7 \pm 9.3 \text { // Control group: } 45.8 \pm 3.2 \\
\text { No statistically significant differences }\end{array}$ \\
\hline $\begin{array}{l}\text { Choukroun et al, } 2006 \\
\text { France [70] }\end{array}$ & $\begin{array}{l}\text { Histomorphometric assessment (\%) } \\
\text { New bone formation: } \\
\text { FDBA + PRF after } 4 \text { months: } 65 \% \text { (mean: } 20.9 \% \text { range: } 18.6-30.3 \% \text { ) } \\
\text { FDBA after } 8 \text { months: } 69 \% \text { (mean: } 20.3 \% \text { range: } 18-23.7 \% \text { ) }\end{array}$ \\
\hline $\begin{array}{l}\text { Comert et al, } \\
2017 \\
\text { Turkey [72] }\end{array}$ & $\begin{array}{l}\text { Histomorphometric assessment }(\%) \text { (mean } \pm \text { standard deviation) } \\
\text { New bone formation: } \\
\text { Group A (P-PRP }+\beta-\mathrm{TCP}): 34.83 \pm 10.1 / / \text { Group B }(\mathrm{PRF}+\beta-\mathrm{TCP}): 32.03 \pm 6.3 / / \text { Control group }(\beta-\mathrm{TCP}): 33.40 \pm 10.4(\mathrm{p}=0.83) \\
\text { Residual graft particle area: } \\
\text { Group A (P-PRP }+\beta-\mathrm{TCP}): 28.98 \pm 7.9 / / \text { Group B }(\mathrm{PRF}+\beta-\mathrm{TCP}): 32.66 \pm 7.5 / / \text { Control group }(\beta-\mathrm{TCP}): 33.39 \pm 10.3(\mathrm{p}=0.69) \\
\text { Soft (fibrous) tissue area: } \\
\text { Group A (P-PRP }+\beta-\mathrm{TCP}): 36.19 \pm 13.9 / / \text { Group B }(\mathrm{PRF}+\beta-\mathrm{TCP}): 35.31 \pm 10.8 / / \text { Control group }(\beta-\mathrm{TCP}): 36.21 \pm 10.6(\mathrm{p}=0.98)\end{array}$ \\
\hline $\begin{array}{l}\text { Del Fabbro et al, } 2013 \\
\text { Italy [73] }\end{array}$ & $\begin{array}{l}\text { Quality of life in the postoperative treatment } \\
\text { Pain (VAS Score): The use of PRGF resulted in significant reduction of the perceived pain during the second and third postoperative day as } \\
\text { compared to the control group. From day } 4 \text {, the mean VAS scores of the two groups were similar. } \\
\text { Patients in the PRGF group reported consistently less swelling, less hematoma and less discomfort regarding chewing and speaking } \\
\text { throughout the evaluation period. } \\
\text { Bleeding was significantly lower in the first } 2 \text { days in the PRGF group }(\mathrm{p}=0.01)\end{array}$ \\
\hline
\end{tabular}


Table 4. Cont.

\begin{tabular}{|c|c|}
\hline $\begin{array}{l}\text { Author, Year } \\
\text { Country }\end{array}$ & Outcome Measures \\
\hline $\begin{array}{l}\text { Gassling et al, } \\
2013 \\
\text { Germany [74] }\end{array}$ & $\begin{array}{l}\text { Histomorphometric assessment (\%) } \\
\text { New (vital) bone formation: } \\
\text { Intervention group (PRF): mean: } 17.0 \% \text { range: } 7.8-27.8 \% \text { // Control group (collagen membrane): mean: } 17.2 \% \text { range: } 8.5-24.2 \% \\
\text { Residual bone-substitute material: } \\
\text { Intervention group (PRF): mean: } 15.9 \% \text { range: } 0.9-33.4 \% \text { // Control group (collagen membrane): mean: } 17.3 \% \text { range: } 0.7-33.5 \%\end{array}$ \\
\hline $\begin{array}{l}\text { Gurler et al, } \\
2016 \\
\text { Turkey [75] }\end{array}$ & $\begin{array}{l}\text { Postoperative complications of direct sinus lifting } \\
\text { Gradual improvements in the L-PRF group regarding postoperative pain, swelling, sleeping, eating, phonetics, activities of daily living and } \\
\text { missed work days, but not statistically significant }(p>0.05) \\
\text { Wound healing in both groups was uneventful } \\
\text { Healing index scores of the L-PRF group were higher than the control group on the } 7^{\text {th }}(4.2 \pm 0.9 \mathrm{vs} .3 .6 \pm 0.7) \text { and } 14^{\text {th }}(4.7 \pm 0.4 \mathrm{vs} .4 .4 \pm \\
0.5) \text { days, but not statistically significant }(\mathrm{p}=0.13, \mathrm{p}=0.19 \text {; respectively) }\end{array}$ \\
\hline $\begin{array}{l}\text { Nizam et al, } \\
2017 \\
\text { Turkey [41] }\end{array}$ & $\begin{array}{l}\text { Histomorphometric assessment }(\%) \text { (mean } \pm \text { standard deviation) } \\
\text { New bone formation: Intervention group (L-PRF): } 21.4 \pm 8.8 / / \text { Control group: } 21.2 \pm 5.6(p=0.96) \\
\text { Residual bone graft: Intervention group (L-PRF): } 25.9 \pm 9.5 / / \text { Control group: } 32.8 \pm 5.9(p=0.06) \\
\text { Bone graft in contact with newly formed bone: Intervention group (L-PRF): } 47.3 \pm 12.3 / / \text { Control group: } 54.0 \pm 8.4(p=0.16) \\
\text { Soft tissue component: Intervention group (L-PRF): } 52.7 \pm 12.5 / / \text { Control group: } 45.9 \pm 8.4(p=0.16) \\
\text { Radiographic assessment }(\mathrm{mm}) \text { (mean } \pm \text { standard deviation) } \\
\text { Augmented bone height: Intervention group (L-PRF): } 13.6 \pm 1.1 / / \text { Control group: } 13.5 \pm 1.2(p=0.88) \\
\text { Implant survival (after } 1 \text { year of follow-up) } \\
\text { 100\% in both groups }\end{array}$ \\
\hline $\begin{array}{l}\text { Olgun et al, } \\
2018 \\
\text { Turkey [45] }\end{array}$ & $\begin{array}{l}\text { Histomorphometric assessment (\%) (mean } \pm \text { standard deviation) } \\
\text { New bone formation: Intervention group (T-PRF): } 16.6 \pm 1.1 / / \text { Control group: } 17.3 \pm 2.5(\mathrm{p}=0.85) \\
\text { Cancellous bone ratio: Intervention group (T-PRF): } 24.0 \pm 1.5 / / \text { Control group: } 22.7 \pm 2.6(\mathrm{p}=0.74) \\
\text { Radiographic assessment (mean } \pm \text { standard deviation or median (IQR)) } \\
\text { Bone height }(\mathrm{mm}): \text { Intervention group (T-PRF): } 11.7 \pm 2.4 / / \text { Control group: } 19.9 \pm 7.4(\mathrm{p}=0.05) \\
\text { Bone volume }\left(\mathrm{mm}^{3}\right) \text { : Intervention group (T-PRF): } 172.7(82.6) / / \text { Control group: } 264.6(70.2)(\mathrm{p}=0.001) \\
\text { Bone density }(\mathrm{hu}): \text { Intervention group (T-PRF): } 86.7(43.6) / / \text { Control group: } 160.8(63.6)(\mathrm{p}=0.001) \\
\text { Control group had 53\% better volume, } 86 \% \text { better density and } 69 \% \text { better height compared to T-PRF group. } \\
\text { Clinical assessment (mean } \pm \text { standard deviation) } \\
\text { Implant stability (Implant stability quotient): Intervention group (T-PRF): } 68.5 \pm 8.9 / / \text { Control group: } 66.4 \pm 8.3(\mathrm{p}=0.61)\end{array}$ \\
\hline
\end{tabular}


Table 4. Cont

\begin{tabular}{|c|c|c|c|}
\hline $\begin{array}{l}\text { Author, Year } \\
\text { Country }\end{array}$ & \multicolumn{3}{|c|}{ Outcome Measures } \\
\hline $\begin{array}{l}\text { Pichotano et al, } \\
2018 \\
\text { Brazil [42] }\end{array}$ & \multicolumn{3}{|c|}{$\begin{array}{l}\text { Radiographic assessment } \\
\left.\text { Graft volume dimensional changes (mean graft reduction between } \mathrm{T}_{1}-\mathrm{T}_{2}{ }^{\mathrm{c}}\right)(\mathrm{mean} \pm \text { standard deviation) } \\
\mathrm{Cm}^{3}=\text { Intervention group (L-PRF): } 0.58 \pm 0.26 / / \text { Control group: } 0.55 \pm 0.34(\mathrm{p}=0.78) \\
\%=\text { Intervention group (L-PRF): } 33.1 \pm 10.7 / / \text { Control group: } 36.7 \pm 15.8(\mathrm{p}=0.47) \\
\text { Histomorphometric assessment (\%) (mean } \pm \text { standard deviation) } \\
\text { New bone formation: Intervention group (L-PRF): } 44.6 \pm 13.9 / / \text { Control group: } 30.0 \pm 8.4(\mathrm{p}=0.008) \\
\text { Residual graft material: Intervention group (L-PRF): } 3.6 \pm 4.2 / / \text { Control group: } 13.7 \pm 9.9(\mathrm{p}=0.01) \\
\text { Soft (fibrous) tissue: Intervention group (L-PRF): } 26.6 \pm 11.1 / / \text { Control group: } 30.6 \pm 12.5(\mathrm{p}=0.38) \\
\text { Clinical assessment } \\
\text { Implant stability (Implant stability quotient) (mean } \pm \text { standard deviation) } \\
\text { After implant placement: Intervention group (L-PRF): } 60.9 \pm 9.3 / / \text { Control group: } 75.1 \pm 5.7(\mathrm{p}<0.001) \\
\text { At implant loading: Intervention group (L-PRF): } 76.1 \pm 5.9 / / \text { Control group: } 75.7 \pm 6.1(\mathrm{p}=0.99) \\
\text { Implant survival (after } 1 \text { year of follow-up): } 100 \% \text { in both groups }\end{array}$} \\
\hline $\begin{array}{l}\text { Tatullo et al, } \\
2012 \\
\text { Italy [76] }\end{array}$ & 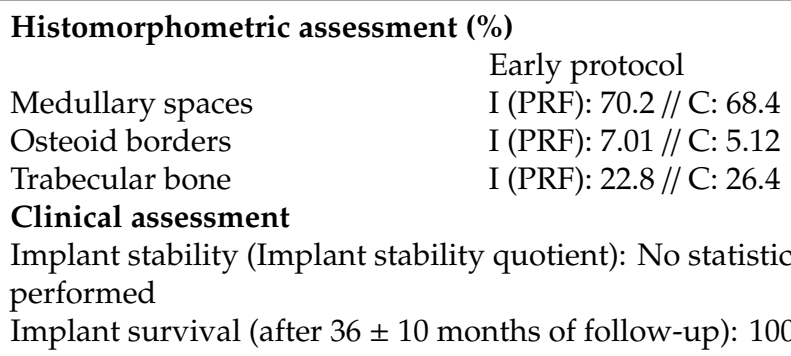 & $\begin{array}{l}\text { Intermediate protocol } \\
\text { I (PRF): } 70.0 \text { // C: } 68.2 \\
\text { I (PRF): } 3.84 \text { // C: } 3.12 \\
\text { I (PRF): } 26.2 \text { // C: } 28.7 \\
\text { rant differences were fou } \\
\text { roups }\end{array}$ & $\begin{array}{l}\text { Late protocol } \\
\text { I (PRF): } 61.4 \text { // C: } 58.2 \\
\text { I (PRF): } 3.5 / / C: 2.9 \\
\text { I (PRF): } 37.1 / / C: 38.9 \\
\text { n groups in each of the protocols }\end{array}$ \\
\hline $\begin{array}{l}\text { Zhang et al, } \\
2012 \\
\text { China [77] }\end{array}$ & $\begin{array}{l}\text { Histomorphometric assessment }(\%)(\text { mean } \pm \text { standard d } \\
\text { New bone formation: Intervention group (PRF): } 18.4 \pm 5 . \\
\text { Residual bone substitute (BioOss): Intervention group (P } \\
\text { Bone-to-bone substitute contact: Intervention group (PRE }\end{array}$ & $\begin{array}{l}\text { group: } 12.9 \pm 5.3(\mathrm{p}=0 \\
6.9 \text { // Control group: } 28.5 \\
6 \text { // Control group: } 18.6\end{array}$ & $\begin{array}{l}=0.14) \\
0.05)\end{array}$ \\
\hline $\begin{array}{l}\text { Abbreviations: } \mathrm{T}_{0}: 10 \mathrm{c} \\
\text { level); IL: distance fron } \\
\text { sinus height (grafted } \mathrm{s} \\
\text { PRF: Plasma-Rich Fibr } \\
\text { Scale; PRGF: Plasma R } \\
\text { that the grafted sinus } \mathrm{c} \\
\text { height): }{ }^{\mathrm{C}} \mathrm{T}_{1} \text { : mean or }\end{array}$ & $\begin{array}{l}\text { r sinus lifting surgery; } \mathrm{T}_{5}: 24 \text { months after prosthetic loading; BL } \\
\text { ex to the head of the fixture (implant length); GSH distance from } \\
\text { htt); OSH: distance from the intraoral marginal bone to the lowe } \\
\text { P: Platelet-Rich Plasma; } \beta \text { - TCP: Beta-Tricalcium Phosphate; L-P } \\
\text { owth Factors; I: Intervention group; C: Control group; a BL/IL ra }\end{array}$ & $\begin{array}{l}\text { the top of the bone-to-im } \\
\text { marginal bone to the graf } \\
\text { original sinus floor (origir } \\
\text {-PRF; T-PRF: Titanium-P } \\
\text { mean change in height of } \\
\text { sinus (values } \geq 1 \text { shows tl }\end{array}$ & $\begin{array}{l}\text { region to the head of the fixture (bone } \\
\text { above the lowest part of the origina } \\
\text { t); FDBA: freeze-dried bone allograft } \\
\text { quartile range; VAS: Visual Analogue } \\
\text { floor for implant (values } \geq 1 \text { indicates } \\
\text { sinus floor is above the original sinus } \\
\text { for control group. }\end{array}$ \\
\hline
\end{tabular}




\subsection{Synthesis of Results}

\subsubsection{Sole Use of Platelet Concentrates in Sinus Floor Augmentation}

i- Radiographic assessment:

Data on bone gain and/or bone height was available for nine studies. Anitua et al. [53] reported a mean bone height of $8.8 \pm 1.4 \mathrm{~mm}$ and a mean bone gain of $4.3 \pm 2.0 \mathrm{~mm}$ using plasma-rich in growth factors (PRGF) as the only graft material after three years of follow-up in an immediate protocol for short implant placement. Mean bone gain in the other studies varied between a minimum of 3.2-3.4 mm [66-68] and a maximum of $10 \mathrm{~mm}[58,60]$. Kanayama et al. [46] reported a statistically significant mean bone gain in sandblasted acid-etched implants compared to hydroxyapatite implants, and Molemans et al. [67] reported a higher mean bone gain $(5.4 \pm 1.5 \mathrm{~mm})$ with the lateral sinus floor elevation approach versus the transalveolar technique $(3.4 \pm 1.2 \mathrm{~mm})$. Two studies additionally reported similar mean bone height values of $11.5 \mathrm{~mm}$ after six months of follow-up [47,65] (Table 1).

ii- Histomorphometric assessment:

Data on the percentage of newly formed bone was available for only one study [58], which reported a mean of $33 \pm 5 \%$ after six months of follow-up (Table 1 ).

iii- Clinical assessment:

Data on implant survival rate was available in eight studies. Six studies reported an implant survival rate $>95 \%$ after different periods of follow-up. The study by Simonpieri et al. [60], with the longest follow-up, reported an implant survival rate of $100 \%$ after six years. Aoki et al. [64] reported an $85.5 \%$ chance of implant survival after a mean follow-up of 3.5 years (range: 1-7 years). In subgroup analyses, the cumulative survival rates were $100 \%$ for the residual bone height $\geq 4 \mathrm{~mm}$ group and $69.6 \%$ for the residual bone height $<4 \mathrm{~mm}$ group $(\mathrm{p}=0.004)$ (Table 1$)$.

\subsubsection{PRF + Grafting Biomaterials in Sinus Floor Augmentation}

i- Radiographic assessment:

Data on radiographic outcomes was available for four studies. Results from Bolukbasi et al. [69] showed that during the study period (2 years), the PRF + Bio-Oss group had less change in the height of grafted sinus floor for implant compared to the Bio-Oss + Bio-Gide group $(\mathrm{p}=0.02)$. Two years after the prosthetic loading, both groups had similar values for the mean change in the height of grafted sinus with a slightly higher value in the intervention group $(4.49 \pm 0.29 \mathrm{~mm}$ versus $4.14 \pm 0.20 \mathrm{~mm})$ (Table 4). Pichotano et al. [42] did not find differences in graft volume dimensional changes between the leukocyte-platelet rich fibrin (L-PRF) + Bio-Oss group and the control group after healing periods of four and eight months, respectively (Table 4). Similarly, Nizam et al. [41] did not find statistically significant differences in the augmented bone height between the test and control group after six months of sinus lift procedure. However, the results from Olgun et al. [45] showed that there was a statistical significant difference in the augmented bone height between the titanium-PRF (T-PRF) group (used as only grafting material) and the Cells \& Tissue Bank Austria (CTBA) allograft, favoring the latter $(11.7 \pm 2.4 \mathrm{~mm}$ versus $19.9 \pm 7.4 \mathrm{~mm}$, respectively). Authors reported that the CTBA allograft group had $53 \%$ better volume, $86 \%$ better density, and $69 \%$ better height compared to the T-PRF group (Table 4). Our pooled analyses yielded no statistically significant difference in the mean augmented bone height between the combination of PRF + grafting biomaterial versus use of a graft material (Figure 2A). 


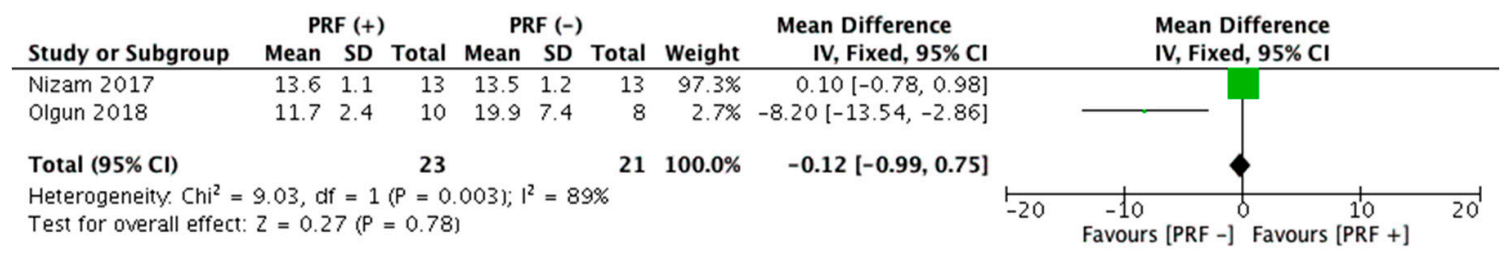

(A)

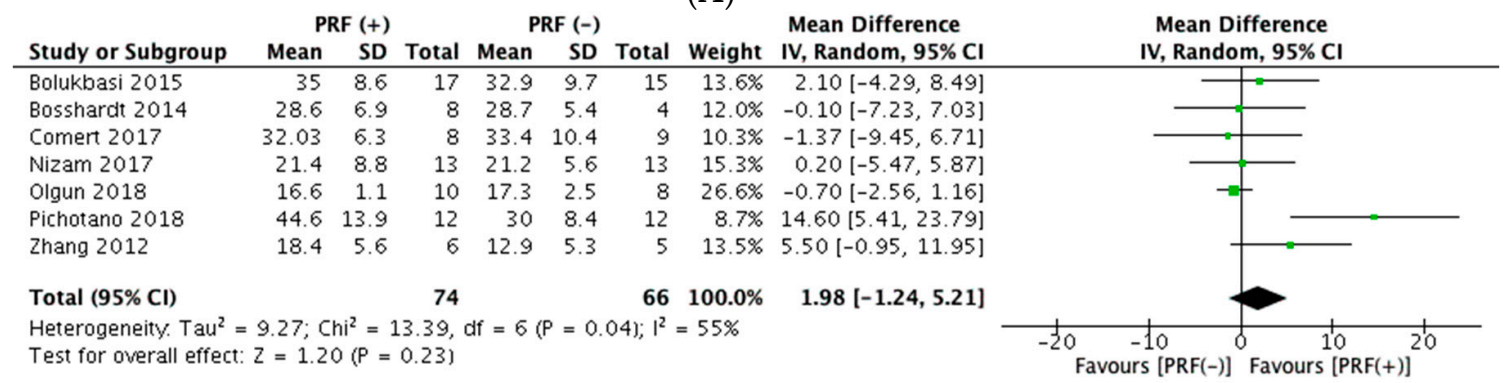

(B)

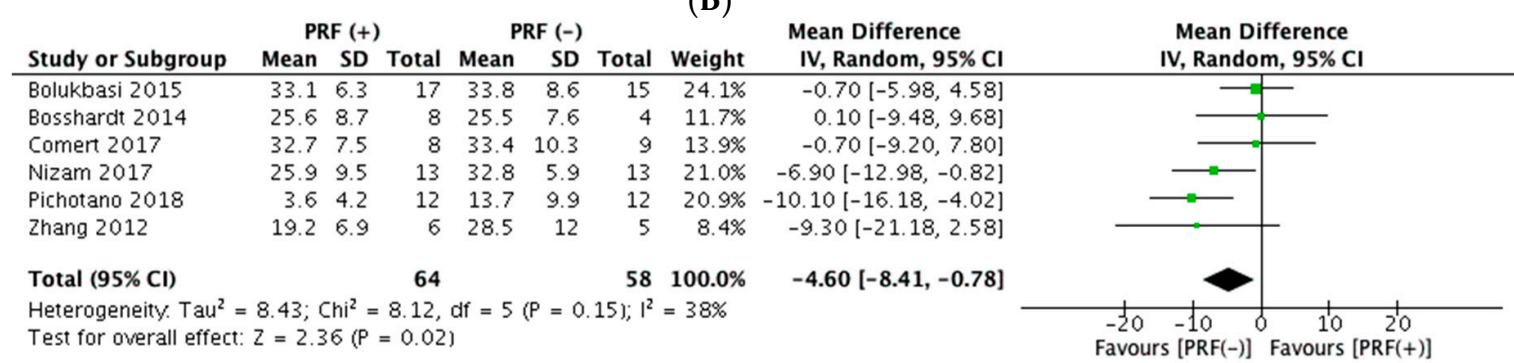

(C)

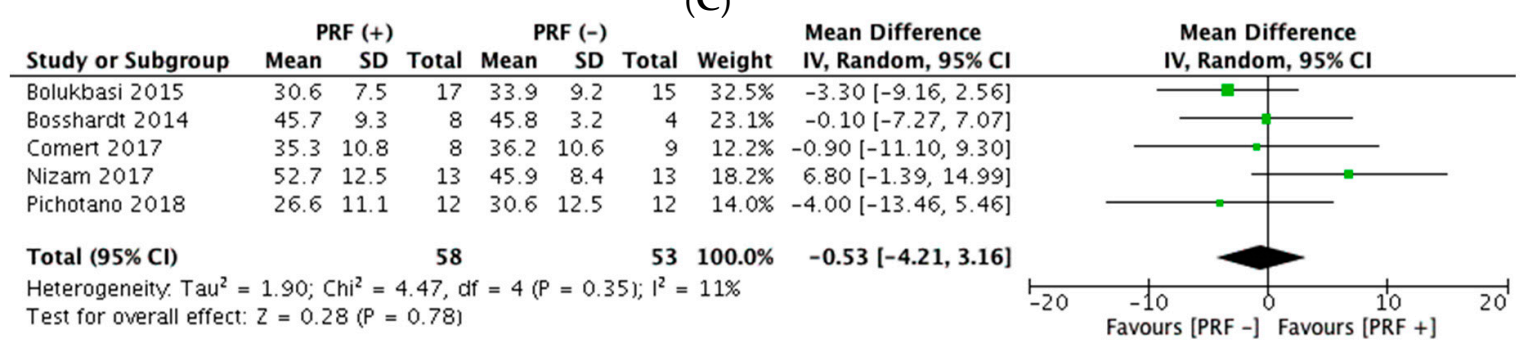

(D)

Figure 2. Plot for comparison: PRF and grafting biomaterial in sinus floor augmentation. (A) Augmented bone height (mm), (B) Percentage of new bone formation, (C) Percentage of residual bone-substitute material, and (D) Percentage of soft-tissue area.

ii- Histomorphometric assessment:

Ten studies provided data on histomorphometric assessment. Regarding the percentage of new bone formation, the majority of the studies reported a similar mean percentage of newly formed bone for both the test and control groups, without a statistically significant difference between them, showing no apparent additional effects of PRF when combined with other grafting biomaterial (Table 4). The percentage of new bone formation varied across studies between $17 \%$ and $45 \%$ in the PRF + grafting biomaterial group and 13\% and 33\% in the grafting biomaterial group. Only Pichotano et al. [42] demonstrated an increased percentage of newly formed bone in the L-PRF + Bio-Oss group compared to the Bio-Oss group ( $44.6 \pm 13.9 \%$ versus $30.0 \pm 8.4 \%$, respectively). Zhang et al. [77] reported that the percentage of new bone formation in the PRF group was 1.4 times higher compared to the control group (Bio-Oss) $(\mathrm{p}=0.14)$. Results from meta-analyses showed that the percentage of new bone formation was $1.98 \%$ higher in the PRF (+) group compared to the PRF (-) group, although these findings were not statistically significant and had moderate heterogeneity (Figure 2B). 
Studies related to the percentage of residual-bone substitute material obtained similar results, with mean values between 3.6\% and 33\% for the PRF + grafting biomaterial group and 13.7\% and $34 \%$ in the grafting biomaterial group (control group) (Table 4). However, Zhang et al. [77] reported a higher mean percentage of residual graft biomaterial in the control group (Bio-Oss) than in the test group (PRF + Bio-Oss) $(28.5 \pm 12.0 \%$ versus $19.2 \pm 6.9 \%$, respectively. Likewise, Pichotano et al. [42] reported a significantly higher percentage residual bone graft material in the Bio-Oss group (13.7 \pm 9.9\%) compared to the L-PRF + Bio-Oss group $(3.6 \pm 4.2 \%)(\mathrm{p}=0.01)$. The pooled analyses showed a statistically significant lower percentage of residual bone substitute material in the PRF (+) group, with $4.60 \%$ less residual bone graft material compared to the PRF (-) group (mean difference: -4.60 , $95 \%$ CI $-8.41,-0.78)$ with no significant heterogeneity $\left(\mathrm{I}^{2}=38 \%\right.$ ) (Figure 2C).

Five studies reported outcomes regarding the soft tissue (fibrous) area in the maxillary sinus cavity. Three of them $[42,69,72]$ showed a slightly higher increase in the amount of soft tissue component in the control group compared to the intervention group (PRF + grafting biomaterial), but differences were not statistically significant (Table 4). Meta-analyses results showed a higher percentage of soft tissue area in the control group than in the PRF (+) group (mean difference: $-0.53,95 \%$ CI $-4.21,3.16$ ), but differences were not statistically significant (Figure 2D).

iii- Clinical assessment:

Data on implant survival and implant stability was available for three studies. Studies reported a $100 \%$ implant survival rate for both the intervention and control groups after one year of follow-up [41,42] and three years of follow-up [76]. Implant stability was assessed through the implant stability quotient (ISQ), and there were no significant differences between compared groups in terms of stability values [45,76]. Pichotano et al. [42] reported higher ISQ values in the control group (75.1 \pm 5.7) compared to the L-PRF group $(60.9 \pm 9.3)$ immediately after implant placement but no statistically significant difference between groups at implant loading ( $\mathrm{p}=0.99$ ) (Table 4).

Gurler et al. [75] showed higher healing index scores in the L-PRF + Minner-Oss group compared to that of the control group (Minner-Oss) on the seventh (4.2 \pm 0.9 versus $3.6 \pm 0.7)$ and $14^{\text {th }}(4.7 \pm 0.4$ versus $4.4 \pm 0.5$ ) day, but there was no statistical significance (Table 4 ).

iv- Patient outcomes:

Two studies provided data on patients' outcomes. Gurler et al. [75] described gradual improvement in the L-PRF group compared to the control group regarding postoperative pain, swelling, sleeping, eating, phonetics, activities of daily living, and missed work days, but the differences were not statistically significant $(\mathrm{p}>0.05)$. Del Fabbro et al. [73] stated that the use of PRGF combined with Bio-Oss resulted in a significant reduction of the perceived pain during the second and third postoperative day as compared to the control group (Bio-Oss). Patients in the test group also reported consistently less swelling, less hematoma, and less discomfort regarding chewing and speaking throughout the evaluation period (one week) (Table 4).

\section{Discussion}

\subsection{Summary of Evidence}

The existing evidence proved to be scarce in terms of the use of only platelet concentrates in maxillary sinus augmentation, with a lack of available studies that compare the use of only platelet concentrates as the grafting material versus other commonly used biomaterials, indicating that the clinical application of PRF in sinus lift procedures is relatively new. The majority of the studies included small cohorts of patients needing sinus floor augmentation with short-term follow-up (6-12 months). Efficacy of the sole use of platelet concentrates could not be assessed given the nature of the study design and lack of a control group. However, results from the studies showed that the use of PRF as the sole filling material during sinus lift with immediate implant placement is a reliable method that could lead to new bone formation and bone gain. The study by Olgun et al. [45] was the only RCT that compared the use of T-PRF versus CTBA allograft. Their results showed no differences in terms of 
new bone formation and implant stability, but radiographic assessment demonstrated that the CTBA allograft group had a statistical significant better bone volume (53\%), bone density (86\%), and height (69\%) compared to the T-PRF group.

The evidence regarding the use of PRF in combination with other grafting biomaterials in maxillary sinus floor augmentation seems to provide no additional beneficial effects in terms of augmented bone height, percentage of soft tissue area, implant survival rate, and implant stability. A statistically significant difference revealed a lower percentage of residual bone substitute material favoring the PRF (+) group when compared to the use of grafting biomaterials alone (mean difference: $-4.60,95 \%$ $\mathrm{CI}-8.41,-0.78)$ with no significant heterogeneity $\left(\mathrm{I}^{2}=38 \%\right)$. In addition, our pooled analyses showed a slightly higher percentage of new bone formation in the PRF group when compared to the use of grafting biomaterials alone. This advantage may be possible due to its osteoinductive properties and the enhanced revascularization process, which allows for improved healing periods of the bone tissue as well. In that context, Choukroun et al. [70] reported no differences in newly formed bone between the PRF + freeze-dried bone allograft (FDBA) protocol versus the FDBA alone protocol, after 4 and 8 months of healing period, respectively, concluding that the addition of PRF could reduce the healing time prior to implant placement [70].

Our results are consistent with other previous systematic reviews [40,78-80] and, to our knowledge, this is the second systematic review to provide pooled analyses. The previous systematic review [40] included five RCTs, so we broadened the search and included 12 studies, from which nine were RCTs. Included studies varied in their methodological quality, the majority of the included studies being assessed as high risk of bias. Only one study [42] was assessed as low risk of bias and reported a statistical high percentage of new bone formation in the L-PRF group compared to the control group $(44.6 \pm 16.9 \%$ versus $30.0 \pm 8.45$, respectively) and no differences between groups regarding graft volume dimensional changes, implant stability, and implant survival rate.

\subsection{Limitations}

Due to the lack of high-quality evidence, results must be interpreted with caution. Most of the included studies failed to specify their methods for allocation concealment, and some did not report the methods for random sequence generation, potentially introducing selection bias. Due to the nature of the intervention of interest, the majority of the studies lack any blinding of participants and personnel introducing, theoretically, performing bias. The degree to which the lack of blinding can affect the outcome assessment depends somewhat on the type of outcome measure. However, most of the studies included in this review assessed objective outcomes. Controlled clinical trials, retrospective, and prospective cohort studies have an inherent higher risk of bias. Most of them failed to control other variables that could influence the results. The majority of the available studies are heterogeneous in terms of intervention, comparison, and outcome measures, hindering direct comparisons on a much larger scale and precluding assessing a precise estimate of the beneficial effects of PRF. Another limitation is the language restriction, as only studies published in English were included in this review.

\subsection{Innovation and Challenges}

Traditionally, autogenous bone has been used in sinus floor augmentation to increase the bone height needed for implant placement. Although it is a predictable and effective technique, it has a high morbidity rate and takes approximately six months to be integrated and substituted by osteoconduction [70], delaying the implant placement and, consequently, extending patient treatment. Autologous graft materials partially account for these inconveniences, avoiding the morbidity associated with the use of autogenous bone. The use of PRF clots is a simple, low-cost, and low-resource technique that could be used as a treatment option for sinus lift procedures, improving the healing period, regeneration, and maturation of bone tissue and thus shortening the implant placement stage. In addition to its osteoinductive property, PRF promotes angiogenesis reduces tissue inflammation and infectious reactions [75], and promotes an immune regulation through the gradual release of 
anti-inflammatory cytokines [81]. These properties and advantages made PRF a promising biomaterial for clinicians and maxillofacial surgeons in different fields of dentistry. Clinical applications of PRF in sinus lift augmentation have recently been investigated. Moreover, there is a lack of evidence provided by RCTs related to the effects of PRF as the sole grafting material in sinus lift procedures with atrophic maxillaries $(<5 \mathrm{~mm})$. Preliminary studies have described positive results in one-stage implant placement protocols regarding bone gain and implant survival, but it is necessary to compare the use of only PRF with the use of other biomaterials or the use of PRF in addition to other biomaterials to assess its effectiveness.

\section{Conclusions}

There is no sufficient robust evidence to make firm conclusions regarding the beneficial effects of the sole use of platelet concentrates in sinus augmentation procedures. Although case series and cohort studies have shown favorable outcomes regarding implant survival, bone gain, and bone height, RCTs are required to assess whether platelet concentrates used as the only grafting materials are at least not inferior to autologous bone or other biomaterials used in sinus floor augmentation. The addition of PRF to other biomaterials appears to provide no further beneficial effects or to improve the outcomes in sinus lift procedures. Due to their biological properties, it could be considered as a reliable treatment option with the advantages of an improved healing period with a consequent earlier implant placement stage, enhanced new bone formation, and improved postoperative complications. Nevertheless, well-designed and conducted RCTs with the same PRF protocol preparation, blinding of personnel in charge of outcome assessment, and long follow-up periods are necessary to confirm the available results, determining their clinical implications and recommendations for the clinical practice.

Author Contributions: Conception: H.O.-M. and J.L.-L.; Validation: E.V.-O., A.E.-D., C.S.-H., R.A.-M., E.V.-O., and J.L.-L.; Formal analysis: C.S.-H. and J.L.-L.; Investigation: H.O.-M.; C.S.-H., and J.L.-L.; Resources: H.O.-M.; C.S.-H., and J.L.-L.; Data synthesis: H.O.-M.; C.S.-H., and J.L.-L.; Writing—original draft preparation: writing - original draft preparation; Writing — review and editing: writing-review and editing; Supervision: R.A.-M., J.L.-L., and E.V.-O.; Project administration: J.L.-L. and E.V.-O. All authors have read and agreed to the published version of the manuscript.

Funding: This research received no external funding.

Conflicts of Interest: The authors declare no potential conflict of interest.

\section{References}

1. Briceño, J.F.; Estrada, J.H. Maxillary Sinus Augmentation: Anatomic and Clinic Considerations. Literature Review. Univ. Odontol. 2012, 31, 27-55.

2. Esposito, M.; Grusovin, M.G.; Felice, P.; Karatzopoulos, G.; Worthington, H.V.; Coulthard, P. The efficacy of horizontal and vertical bone augmentation procedures for dental implants - a Cochrane systematic review. Eur. J. Oral Implantol. 2009, 2, 167-184. [PubMed]

3. Monje, A.; Urban, I.; Miron, R.; Caballe-Serrano, J.; Buser, D.; Wang, H. Morphologic patterns of the atrophic posterior maxilla and clinical implications for bone regenerative therapy. Int. J. Periodontics Restorative Den. 2017, 37, e279-e289. [CrossRef] [PubMed]

4. Tan, W.C.; Lang, N.P.; Zwahlen, M.; Pjetursson, B.E. A systematic review of the success of sinus floor elevation and survival of implants inserted in combination with sinus floor elevation. Part II: transalveolar technique. J. Clin. Periodontol. 2008, 35, 241-254. [CrossRef] [PubMed]

5. Zijderveld, S.A.; Van den Bergh, J.P.; Schulten, E.A.; Bruggenkate, C.M. Anatomical and surgical findings and complications in 100 consecutive maxillary sinus floor elevation procedures. J. Oral Maxillofac. Surg. 2008, 66, 1426-1438. [CrossRef] [PubMed]

6. Boyne, P.J.; James, R.A. Grafting of the maxillary sinus floor with autogenous marrow and bone. J. Oral Surg. 1980, 38, 613-616.

7. Tatum, H., Jr. Maxillary and sinus implant reconstructions. Dent. Clin. North Am. 1986, 30, 207-229. 
8. Pjetursson, B.E.; Tan, W.C.; Zwahlen, M.; Lang, N.P. A systematic review of the success of sinus floor elevation and survival of implants inserted in combination with sinus floor elevation: part I: lateral approach. J. Clin. Periodontol. 2008, 35, 216-240. [CrossRef]

9. Thoma, D.S.; Cha, J.K.; Jung, U.W. Treatment concepts for the posterior maxilla and mandible: short implants versus long implants in augmented bone. J. Periodontal Implant Sci. 2017, 47, 2-12. [CrossRef]

10. Mohan, N.; Wolf, J.; Dym, H. Maxillary sinus augmentation. Dent. Clin. North Am. 2015, 59, 375-388. [CrossRef]

11. Al-Moraissi, E.A.; Altairi, N.H.; Abotaleb, B.; Al-Iryani, G.; Halboub, E.; Alakhali, M.S. What is the most effective rehabilitation method for posterior maxillas with 4 to $8 \mathrm{~mm}$ of residual alveolar bone height below the maxillary sinus with implant-supported prostheses? A frequentist network meta-analysis. J. Oral Maxillofac. Surg. 2019, 77, 70.e1-70.e33. [CrossRef] [PubMed]

12. Santoro, M.; Pippi, R. Intrasinus bone gain with the osteotome sinus floor elevation technique: a review of the literature. Int. J. Oral Maxillofac. Implants 2018, 33, 995-1002. [CrossRef] [PubMed]

13. Carreño-Carreño, J.; Aguilar-Salvatierra, A.; Gómez-Moreno, G.; García-Carreño, E.M.; Menéndez López-Mateos, M.L.; Perrotti, V.; Piattelli, A.; Calvo-Guirado, J.L.; Menéndez-Núñez, M. Update of surgical techniques for maxillary sinus augmentation: a systematic literature review. Implant Dent. 2016, 25, 839-844. [CrossRef] [PubMed]

14. Lundgren, S.; Cricchio, G.; Hallman, M.; Jungner, M.; Rasmusson, L.; Sennerby, L. Sinus floor elevation procedures to enable implant placement and integration: techniques, biological aspects and clinical outcomes. Periodontology 2000 2017, 73, 103-120. [CrossRef] [PubMed]

15. Summers, R.B. The osteotome technique: part3-less invasive methods of elevating the sinus floor. Compendium 1994, 15, 698, 702-704. [PubMed]

16. Ting, M.; Rice, J.G.; Braid, S.M.; Lee, C.Y.; Suzuki, J.B. Maxillary sinus augmentation for dental implant rehabilitation of the edentulous ridge: a comprehensive overview of systematic reviews. Implant Dent. 2017, 26, 438-464. [CrossRef]

17. Lundgren, S.; Andersson, S; Gualini, F; Sennerby, L. Bone reformation with sinus membrane elevation: a new surgical technique for maxillary sinus floor augmentation. Clin Implant Dent Relat Res. 2004, 6, 165-173. [CrossRef]

18. Duan, D.H.; Fu, J.H.; Qi, W.; Du, Y.; Pan, J.; Wang, H.L. Graft-free maxillary sinus floor elevation: A systematic review and meta-analysis. J. Periodontol. 2017, 88, 550-564. [CrossRef]

19. Starch-Jensen, T.; Schou, S. Maxillary sinus membrane elevation with simultaneous installation of implants without the use of a graft material: a systematic review. Implant Dent. 2017, 26, 621-633. [CrossRef]

20. Moraschini, V.; Uzeda, M.G.; Sartoretto, S.C.; Calasans-Maia, M.D. Maxillary sinus floor elevation with simultaneous implant placement without grafting materials: a systematic review and meta-analysis. Int. J. Oral Maxillofac. Surg. 2017, 46, 636-647. [CrossRef]

21. Danesh-Sani, S.A.; Loomer, P.M.; Wallace, S.S. A comprehensive clinical review of maxillary sinus floor elevation: anatomy, techniques, biomaterials and complications. Br. J. Oral Maxillofac. Surg. 2016, 54, 724-730. [CrossRef] [PubMed]

22. Starch-Jensen, T.; Jensen, J.D. Maxillary sinus floor augmentation: a review of selected treatment modalities. J. Oral Maxillofac. Res. 2017, 8, e3. [CrossRef] [PubMed]

23. Jeong, K.I.; Kim, S.G.; Kim, Y.K.; Oh, J.S.; Jeong, M.A.; Park, J.J. Clinical study of graft materials using autogenous teeth in maxillary sinus augmentation. Implant Dent. 2011, 20,471-475. [CrossRef] [PubMed]

24. Roselló-LLabres, X.; Camps, A.R.; Jané-Salas, E.; Albuquerque, R.; Velasco-Ortega, E.; López-López, J. Graft materials in oral surgery: revision. J. Biomim. Biomater. Tissue Eng. 2014, 19, 124. [CrossRef]

25. Gual-Vaqués, P.; Polis-Yanes, C.; Estrugo-Devesa, A.; Ayuso-Montero, R.; Marí-Roig, A.; López-López, J. Autogenous teeth used for bone grafting: a systematic review. Med. Oral Patol Oral Cir. Bucal. 2018, 23, e112-e119. [CrossRef] [PubMed]

26. Clavero, J.; Lundgren, S. Ramus or chin grafts for maxillary sinus inlay and local onlay augmentation: comparison of donor site morbidity and complications. Clin. Implant Dent. Relat. Res. 2003, 5, 154-160. [CrossRef]

27. Shanbhag, S.; Shanbhag, V.; Stavropoulos, A. Volume changes of maxillary sinus augmentation over time: a systematic review. Int. J. Oral Maxillofac. Implants 2014, 29, 881-892. [CrossRef] 
28. Nkenke, E.; Neukam, F. Autogenous bone harvesting and grafting in advanced jaw resorption: morbidity, resorption and implant survival. Eur. J. Oral. Implantol. 2014, 7 (Suppl. S2), S203-S217.

29. Jensen, T.; Schou, S.; Stavropoulos, A.; Terheyden, H.; Holmstrup, P. Maxillary sinus floor augmentation with Bio-Oss or Bio-Oss mixed with autogenous bone as graft in animals: a systematic review. Int. J. Oral Maxillofac. Surg. 2012, 41, 114-120. [CrossRef]

30. Aludden, H.C.; Mordenfeld, A.; Hallman, M.; Dahlin, C.; Jensen, T. Lateral ridge augmentation with Bio-Oss alone or Bio-Oss mixed with particulate autogenous bone graft: a systematic review. Int. J. Oral Maxillofac. Surg. 2017, 46, 1030-1038. [CrossRef]

31. Esposito, M.; Felice, P.; Worthington, H.V. Interventions for replacing missing teeth: augmentation procedures of the maxillary sinus. Cochrane Database Syst. Rev. 2014, 13, CD008397. [CrossRef] [PubMed]

32. Aghaloo, T.L.; Misch, C.; Lin, G.H.; Iacono, V.J.; Wang, H.L. Bone augmentation of the edentulous maxilla for implant placement: a systematic review. Int. J. Oral Maxillofac. Implants 2016, 31, 19-30. [CrossRef] [PubMed]

33. Danesh-Sani, S.A.; Engebretson, S.P.; Janal, M.N. Histomorphometric results of different grafting materials and effect of healing time on bone maturation after sinus floor augmentation: a systematic review and meta-analysis. J. Periodontal Res. 2017, 52, 301-312. [CrossRef] [PubMed]

34. Starch-Jensen, T.; Aludden, H.; Hallman, M.; Dahlin, C.; Christensen, A.E.; Mordenfeld, A. A systematic review and meta-analysis of long-term studies (five or more years) assessing maxillary sinus floor augmentation. Int. J. Oral Maxillofac. Surg. 2017, 22, 103-116. [CrossRef] [PubMed]

35. Whitman, D.H.; Berry, R.L.; Green, D.M. Platelet gel: an autologous alternative to fibrin glue with applications in oral maxillofacial surgery. J Oral Maxillofac. Surg. 1997, 55, 1294-1299. [CrossRef]

36. Dohan, D.M.; Rasmusson, L.; Albrektsson, T. Classification of platelet concentrates: from pure platelet-rich plasma (P-PRP) to leucocyte- and platelet-rich fibrin (L-PRF). Trends Biotechnol. 2009, 27, 158-167. [CrossRef]

37. Schliephake, H.; Sicilia, A.; Nawas, B.A.; Donos, N.; Gruber, R.; Jepsen, S.; Milinkovic, I.; Mombelli, A.; Navarro, J.M.; Quirynen, M.; et al. Drugs and diseases: summary and consensus statements of group 1. The 5th EAO consensus conference 2018. Clin. Oral Implants Res. 2018, 29, 93-99. [CrossRef]

38. Chieruzzi, M.; Pagano, S.; Moretti, S.; Pinna, R.; Milia, E.; Torre, L.; Eramo, S. Nanomateriasl for tissue engineering in dentistry. Nanomaterials 2016, 6, 134. [CrossRef]

39. Strauss, F.J.; Stähli, A.; Gruber, R. The use of platelet-rich fibrin to enhance the outcomes of implants therapy: a systematic review. Clin. Oral Implants Res. 2018, 29, 6-19. [CrossRef]

40. Liu, R.; Yan, M.; Chen, S.; Huang, W.; Wu, D.; Chen, J. Effectiveness of platelet-rich fibrin as an adjunctive material to bone graft in maxillary sinus augmentation: a meta-analysis of randomized controlled trails. Biomed. Res. Int. 2019, 2019, 1-10. [CrossRef]

41. Nizam, N.; Eren, G.; Akcalı, A.; Donos, N. Maxillary sinus augmentation with leukocyte and platelet-rich fibrin and deproteinized bovine bone mineral: a split-mouth histological and histomorphometric study. Clin. Oral Implants Res. 2018, 29, 67-75. [CrossRef]

42. Pichotano, E.C.; de Molon, R.S.; de Souza, R.V.; Austin, R.S.; Marcantonio, E.; Zandim-Barcelos, D.L. Evaluation of L-PRF combined with deproteinized bovine bone mineral for early implant placement after maxillary sinus augmentation: a randomized clinical trial. Clin. Implant Dent. Relat. Res. 2019, 21, 253-262. [CrossRef] [PubMed]

43. Roldán, C.J.; Jepsen, S.; Schmidt, C.; Knuppel, H.; Rueger, D.C.; Acil, Y.; Terheyden, H. Sinus floor augmentation with simultaneous placement of dental implants in the presence of platelet-rich plasma or recombinant human bone morphogenetic protein-7. Clin. Oral Impl. Res. 2004, 15, 716-723. [CrossRef] [PubMed]

44. Jeong, S.M.; Lee, C.U.; Son, J.S.; Oh, J.H.; Fang, Y.; Choi, B.H. Simultaneous sinus lift and implantation using platelet-rich fibrin as sole grafting material. J. Craniomaxillofac. Surg. 2014, 42, 990-994. [CrossRef] [PubMed]

45. Olgun, E.; Ozkan, S.Y.; Atmaca, H.T.; Yalim, M.; Hendek, M.K. Comparison of the clinical, radiographic, and histological effects of titanium-prepared platelet rich fibrin to allograft materials in sinus-lifting procedures. J. Investig. Clin. Dent. 2018, 9, e12347. [CrossRef]

46. Kanayama, T.; Horii, K.; Senga, Y.; Shibuya, Y. Crestal approach to sinus floor elevation for atrophic maxilla using platelet-rich fibrin as the only grafting material: a 1-Year prospective study. Implant Dent. 2016, 25, 32-38. [CrossRef] 
47. Tajima, N.; Ohba, S.; Sawase, T.; Asahina, I. Evaluation of sinus floor augmentation with simultaneous implant placement using platelet-rich fibrin as sole grafting material. Int. J. Oral Maxillofac. Implants 2013, 28, 77-83. [CrossRef]

48. Ocak, H.; Kutuk, N.; Demetoglu, U.; Balcioglu, E.; Ozdamar, S.; Alkan, A. Comparison bovine bone-autogenic bone misxture versus platelet-rich fibrin for maxillary sinus grafting: histological and histomorphological study. J. Oral Implantol. 2017, 43, 194-201. [CrossRef]

49. Aoki, N.; Kanayama, T.; Maeda, M.; Horii, K.; Miyamoto, H.; Wada, K.; Ojima, Y.; Tsuchimochi, T.; Shibuya, Y. Sinus augmentation by platelet-rich fibrin alone: a report of two cases with histological examinations. Case Rep. Dent. 2016, 2016, 2654645. [CrossRef]

50. Wang, H.; Wang, J.; Guo, T.; Ding, X.; Wanqi, Y.; Zhao, J.; Zhou, Y. The endoscopically assisted transcrestal sinus floor elevation with platelet-rich fibrin at an immediate implantation of periapical lesion site: A case report. Medicine (Baltimore) 2019, 98, e16251. [CrossRef]

51. Zhao, J.H.; Chung, H.T.; Yu, C.C. Clinical application of platelet-rich fibrin as the sole grafting material in maxillary sinus augmentation. J. Formos Med. Assoc. 2015, 114, 779-780. [CrossRef] [PubMed]

52. Anitua, E.; Prado, R.; Orive, G. A lateral approach for sinus elevation using PRGF technology. Clin. Implant Den. Relat. Res. 2009, 11, 23-31. [CrossRef]

53. Anitua, E.; Flores, J.; Alkhraisat, M.H. Transcrestal sinus lift using platelet concentrates in association to short implant placement: a retrospective study of augmented bone height remodeling. Clin. Implant Den. Relat. Res. 2016, 18, 993-1002. [CrossRef] [PubMed]

54. Barbu, H.M.; Andreescu, C.F.; Comaneanu, M.R.; Referendaru, D.; Mijiritsky, E. Maxillary sinus floor augmentation to enable one-stage implant placement by using bovine bone substitute and platelet-rich fibrin. BioMed. Res. Int. 2018, 2018, 6562958. [CrossRef] [PubMed]

55. Inchingolo, F.; Tatullo, M.; Marrelli, M.; Inchingolo, A.M.; Scacco, S.; Inchingolo, A.D.; Dipalma, G.; Vermesan, D.; Abbinante, A.; Cagiano, R. Trial with platelet-rich fibrin and Bio-Oss used as grafting materials in the treatment of the severe maxillar bone atrophy: Clinical and radiological evaluations. EuR. Rev. Med. Pharmacol Sci. 2010, 14, 1075-1084. [PubMed]

56. Khouly, I.; Pardiñas-López, S.; Aliaga, I.; Froum, S. Long-term implant survival after 100 maxillary sinus augmentations using plasma rich in growth factors. Implant. Dent. 2017, 26, 199-208. [CrossRef] [PubMed]

57. Kumar, M. Direct maxillary sinus floor augmentation for simultaneous dental implant placement. Ann. Maxillofac. Surg. 2018, 8, 188-192. [CrossRef]

58. Mazor, Z.; Horowitz, R.A.; Del Corso, M.; Prasad, H.S.; Rohrer, M.D.; Ehrenfest, D. Sinus floor augmentation with simultaneous implant placement using Choukroun's platelet-rich fibrin as the sole mrafting Material: A radiologic and histologic study at 6 months. J. Periodontol. 2009, 80, 2056-2064. [CrossRef]

59. Öncü, E.; Kaymaz, E. Assessment of the effectiveness of platelet rich fibrin in the treatment of schneiderian membrane perforation. Clin. Implant Dent. Relat. Res. 2017, 19, 1009-1014. [CrossRef]

60. Simonpieri, A.; Choukroun, J.; Del Corso, M.; Sammartino, G.; Ehrenfest, D. Simultaneous sinus-lift and implantation using microthreaded implants and leukocyte- and platelet-rich fibrin as sole grafting material: A six-year experience. Implant Dent. 2011, 20, 2-12. [CrossRef]

61. Taschieri, S.; Del Fabbro, M. Postextraction osteotome sinus floor elevation technique using plasma-rich growth factors. Implant Dent. 2011, 20, 418-424. [CrossRef] [PubMed]

62. Badr, M.; Oliver, R.; Pemberton, P.; Coulthard, P. Platelet-rich plasma in grafted maxillae: growth factor quantification and dynamic histomorphometric evaluation. Implant Dent. 2016, 25, 492-498. [CrossRef] [PubMed]

63. Khairy, N.M.; Shendy, E.E.; Askar, N.A.; El-Rouby, D.H. Effect of platelet rich plasma on bone regeneration in maxillary sinus augmentation (randomized clinical trial). Int. J. Oral Maxillofac. Surg. 2013, 42, 249-255. [CrossRef] [PubMed]

64. Aoki, N.; Maeda, M.; Kurata, M.; Hirose, M.; Ojima, Y.; Wada, K.; Shibuya, Y. Sinus floor elevation with platelet-rich fibrin alone: A clinical retrospective study of 1-7 years. J. Clin. Exp. Dent. 2018, 10, e984-e991. [CrossRef]

65. Gülşen, U.; Dereci, O. Evaluation of new bone formation in sinus floor augmentation with injectable platelet-rich fibrin-soaked collagen plug: A pilot study. Implant Dentistry 2019, 28, 220-225. [CrossRef] 
66. Diss, A.; Dohan, D.M.; Mouhyi, J.; Mahler, P. Osteotome sinus floor elevation using Choukroun's platelet-rich fibrin as grafting material: A 1-year prospective pilot study with microthreaded implants. Oral Surg. Oral Med. Oral Pathol. Oral Radiol. Endod. 2008, 105, 572-579. [CrossRef]

67. Molemans, B.; Cortellini, S.; Jacobs, R.; Teughels, W.; Pinto, Quirynen, M. Simultaneous sinus floor elevation and implant placement using leukocyte- and platelet-rich fibrin as a sole graft material. Int. J. Oral Maxillofac. Implants 2019, 34, 1195-1201. [CrossRef]

68. Toffler, M.; Toscano, N.; Holtzclaw, D. Osteotome-mediated sinus floor elevation using only platelet-rich fibrin: an early report on 110 patients. Implant Dent. 2010, 19, 447-456. [CrossRef]

69. Bolukbasi, N.; Ersanli, S.; Keklikoglu, N.; Basegmez, C.; Ozdemir, T. Sinus augmentation with platelet-rich fibrin in combination with bovine bone graft versus bovine bone graft in combination with collagen membrane. J. Oral Implantol. 2015, 41, 586-595. [CrossRef]

70. Choukroun, J.; Diss, A.; Simonpieri, A.; Girard, M.O.; Schoeffler, C.; Dohan, S.; Dohan, A.J.J.; Mouhyi, J.; Dohan, D.M. Platelet-rich fibrin (PRF): a second-generation platelet concentrate. Part V: histologic evaluations of PRF effects on bone allograft maturation in sinus lift. Oral Surg. Oral Med. Oral Pathol, Oral Radiol Endodon. 2006, 101, 299-303. [CrossRef]

71. Bosshardt, D.D.; Bornstein, M.M.; Carrel, J.P.; Buser, D.; Bernard, J.P. Maxillary sinus grafting with a synthetic, nanocrystalline hydroxyapatite-silica gel in humans: histologic and histomorphometric results. Int. J. Periodontics Restorative Dent. 2014, 34, 259-267. [CrossRef] [PubMed]

72. Cömert, K.S.; Güngörmüş, M.; Parlak, S.N. Histologic and histomorphometric assessment of sinus-floor augmentation with beta-tricalcium phosphate alone or in combination with pure-platelet-rich plasma or platelet-rich fibrin: a randomized clinical trial. Clin. Implant Dent. Relat. Res. 2017, 19, 959-967. [CrossRef]

73. Del Fabbro, M.; Corbella, S.; Ceresoli, V.; Ceci, C.; Taschieri, S. Plasma rich in growth factors improves patients' postoperative quality of life in maxillary sinus floor augmentation: preliminary results of a randomized clinical study. Clin. Implant Dent. Relat. Res. 2015, 17, 708-716. [CrossRef] [PubMed]

74. Gassling, V.; Nicolai, P.; Braesen, J.H.; Will, M.; Gierloff, M.; Behrens, E.; Açil, Y.; Wiltfang, J. Comparison of two different absorbable membranes for the coverage of lateral osteotomy sites in maxillary sinus augmentation: a preliminary study. J. Cranio-maxillofac. Surg. 2013, 41, 76-82. [CrossRef] [PubMed]

75. Gurler, G.; Delilbasi, C. Effects of leukocyte-platelet rich fibrin (L-PRF) on post-operative complications of direct sinus lifting. Minerva Stomatol. 2016, 65, 207-212. [PubMed]

76. Tatullo, M.; Marrelli, M.; Cassetta, M.; Pacifici, A.; Stefanelli, L.; Scacco, S.; Dipalma, G.; Pacifici, L.; Inchingolo, F. Platelet rich fibrin (P.R.F.) in reconstructive surgery of atrophied maxillary bones: Clinical and histological evaluations. Int. J. Med. Sci. 2012, 9, 872-880. [CrossRef]

77. Zhang, Y.; Tangl, S.; Huber, C.D.; Lin, Y.; Qiu, L.; Rausch-Fan, X. Effects of Choukroun's platelet-rich fibrin on bone regeneration in combination with deproteinized bovine bone mineral in maxillary sinus augmentation: A histological and histomorphometric study. J. Craniomaxillofac Surg. 2012, 40, 321-328. [CrossRef]

78. Ali, S.; Bakry, S.A.; Abd-Elhakam, H. Platelet rich fibrin in maxillary sinus augmentation: a systematic review. J. Oral Implantol. 2015, 41, 746-753. [CrossRef]

79. Castro, A.B.; Meschi, N.; Temmerman, A.; Pinto, N.; Lambrechts, P.; Teughels, W.; Quirynen, M. Regenerative potential of leucocyte- and platelet-rich fibrin. Part B: sinus floor elevation, alveolar ridge preservation and implant therapy. A systematic review. J. Clinical Periodontol. 2017, 44, 225-234. [CrossRef]

80. Dragonas, P.; Katsaros, T.; Avila-Ortiz, G.; Chambrone, L.; Schiavo, J.H.; Palaiologou, A. Effects of leukocyte-platelet-rich fibrin (L-PRF) in different intraoral bone grafting procedures: a systematic review. Int. J. Oral Maxillofac. Surg. 2019, 48, 250-262. [CrossRef]

81. Dohan, D.M.; Choukroun, J.; Diss, A.; Dohan, S.L.; Dohan, A.J.; Mouhyi, J.; Gogly, B. Platelet-rich fibrin (PRF): a second generation platelet concentrate. Part III: leucocyte activation: a new feature for platelet concentrates? Oral Surg. Oral Med. Oral Pathol. Oral Radiol. Endod. 2006, 101, e51-e55. [CrossRef] [PubMed]

(C) 2020 by the authors. Licensee MDPI, Basel, Switzerland. This article is an open access article distributed under the terms and conditions of the Creative Commons Attribution (CC BY) license (http://creativecommons.org/licenses/by/4.0/). 